\title{
Curvature dependence of quantum gravity
}

\author{
Nicolai Christiansen, ${ }^{1}$ Kevin Falls, ${ }^{1}$ Jan M. Pawlowski, ${ }^{1,2}$ and Manuel Reichert ${ }^{1}$ \\ ${ }^{1}$ Institut für Theoretische Physik, Universität Heidelberg, \\ Philosophenweg 16, 69120 Heidelberg, Germany \\ ${ }^{2}$ ExtreMe Matter Institute EMMI, GSI Helmholtzzentrum für Schwerionenforschung mbH, \\ Planckstrasse 1, 64291 Darmstadt, Germany
}

(Received 7 December 2017; published 15 February 2018)

\begin{abstract}
We investigate the phase diagram of quantum gravity with a vertex expansion about constantly-curved backgrounds. The graviton two- and three-point function are evaluated with a spectral sum on a sphere. We obtain, for the first time, curvature-dependent UV fixed point functions of the dynamical fluctuation couplings $g^{*}(R), \mu^{*}(R)$, and $\lambda_{3}^{*}(R)$, and the background $f(R)$-potential. Based on these fixed point functions we compute solutions to the quantum and the background equation of motion with and without standard model matter. We have checked that the solutions are robust against changes of the truncation.
\end{abstract}

DOI: 10.1103/PhysRevD.97.046007

\section{INTRODUCTION}

Modern theoretical physics is built upon two pillars, namely quantum field theory and general relativity. Theories of quantum gravity aim at the unification of gravity with quantum dynamics. A candidate for a quantum theory of gravity is the asymptotic safety scenario, which goes back to Weinberg's idea in 1976 [1]. Its construction is based on a nontrivial ultraviolet fixed point in the renormalization group flow. The fixed point of asymptotic safety implies coupling constants that are finite at arbitrarily high energy scales, while they depend only on a finite number of free parameters. Hence, an asymptotically safe quantum field theory does not necessarily have a scale of maximal validity and thus can potentially describe physical interactions at the most fundamental level. The possibility of an interacting ultraviolet fixed point in quantum gravity attracted increasing attention over the last two decades. Beginning with the pioneering work by Reuter [2], good evidence for its existence was found in pure gravity setups as well as in systems with gravity coupled to gauge and matter fields [3-70]. For reviews see [71-76].

Most studies on asymptotically safe quantum gravity are based on the functional renormalization group (FRG), [77] and $[78,79]$. In its modern form as a flow equation for the effective action $\Gamma[\phi]$ of the theory it constitutes a powerful method for nonperturbative calculations in continuum quantum field theory. Here $\phi$ is a superfield that comprises

Published by the American Physical Society under the terms of the Creative Commons Attribution 4.0 International license. Further distribution of this work must maintain attribution to the author(s) and the published article's title, journal citation, and DOI. Funded by SCOAP ${ }^{3}$. all fields in the theory. This formulation, as all formulations based on metric correlation functions, demands the introduction of a background metric $\bar{g}_{\mu \nu}$ and a corresponding fluctuation field $h_{\mu \nu}$. Inevitably, correlation functions as well as the effective action depend separately on these fields. Note however, that it is the correlation functions of the fluctuation field that carry the dynamics of the system. Indeed, the flow equation for the effective action is directly proportional to the two-point function (propagator) of the fluctuation field in a general background. Phrased differently, the solution of the flow equation requires the knowledge of two-point and higher correlation functions of the fluctuation field. This already entails that the correlation functions of the background field and mixed correlations of background and fluctuation can only be constructed on the basis of the pure fluctuation field correlations. More details on these important relation and a brief overview of the current state is provided in Sec. II.

Our setup is detailed in Sec. III A and allows for the computation and the distinction of the background and quantum equation of motion (EoM). We argue that these equations have a common solution at a vanishing infrared FRG cutoff scale $k=0$ due to background independence. In turn, the solutions to the background and quantum EoM do not agree at a finite cutoff scale $k \neq 0$, which signals the loss of background independence in the presence of the FRG-regulator. This is also seen in our explicit computations at the ultraviolet fixed point. We further argue that the quantum EoM, and not the background EoM, should be used to determine the self-consistent background at finite $k$.

Solutions to the background EoM appear as minimum in the background potential $f(R) / R^{2}$, which we compute for the first time from the dynamical backgrounddependent fluctuation couplings without a background 
field approximation. In the present work we compute the ultraviolet fixed point background potential $f^{*}(R)$. Interestingly, in the pure quantum gravity setting we do not find a solution to the background EoM, while a solution appears at small positive curvature for standard model matter content. The quantum EoM on the other hand has a solution also in the pure quantum gravity setting.

This work is organized as follows: In Sec. II we discuss the importance of background independence and its manifestation in the current framework. This includes a brief overview and description of the results obtained in the literature. In Sec. III A we introduce the FRG with a particular focus on the background and quantum EoM and the Nielsen identity that relates them. We furthermore introduce the vertex expansion used in this work. In Sec. IV we construct an approximate momentum space on spherical curved backgrounds. This allows us to use previously developed techniques that were based on running correlation functions in momentum space. In Sec. V we present our results, which include the nontrivial ultraviolet fixed point functions for the dynamical couplings as well as a detailed discussion of the background and quantum EoM. In Sec. VI we summarize our results. The technical details are specified in the Appendices.

\section{BACKGROUND INDEPENDENCE IN QUANTUM GRAVITY}

Most applications of the FRG to quantum gravity to date do not resolve the difference between background and fluctuation field and employ the background field approximation. There only one metric $g_{\mu \nu}=\bar{g}_{\mu \nu}+h_{\mu \nu}$ is used in the effective action. However, the non-trivial interplay of the metric fluctuations with the background plays a decisive role for background independence of the theory. These nontrivial relations are governed by nontrivial splitWard or Nielsen identities (NIs), see e.g. [15-17,80-88] for formal progress and applications in scalar theories, gauge theories and gravity. Accordingly, the background field approximation violates the NIs, which leads to the seemingly contradictory situation that it is at odds with background independence even though it only features one metric. In the past decade quite some progress has been made in overcoming the background field approximation, see [3-21,80-88].

\section{A. Approaches to fluctuation and background correlation functions}

All these works should be seen in the context of gaining background independence and physical diffeomorphism invariance in asymptotically safe gravity. Here we briefly summarize the state of the art within the different approaches.

(1) One approach utilizes the fact that the NIs relate background metric correlations to fluctuation ones. This leaves us with a system of one type of correlations and it is possible to solve the system of flow equations for fluctuation correlation functions either directly or implicitly. This strategy has been set up and pursued in [15-17,80-88] for generic theories within the background field approach. At present, applications in gravity still utilize the background field approximation beyond either the first order, or the second order in the fluctuation field [15]. Such a closure of the flow equation with the background field approximation is mandatory and all approaches aim at introducing this approximation on a high order of the fluctuation field. Note in this context that it is only the second and higher order $n$ point functions of the fluctuation field that drive the flow.

(2a) A second approach utilizes the fact that the dynamics of the system is carried by the correlation functions of the fluctuation field. This is also reflected by the fact that the system of flow equations for the fluctuation correlations is closed. Consequently one may solve these flows for a specific background metric that facilitates the computation, e.g. the flat background. Then, background correlations are computed within an expansion or extension about the flat background in order to access the physical background that solves the quantum EoM. This strategy has been set up and pursued in [3-8,10-14] for gravity, also guided by successful applications in nonAbelian gauge theories, see e.g. [89-93]. At present, fluctuation correlations up to the four-point function have been included [11], as well as a full fluctuation effective potential [14]. First results in a Taylor expansion of the background about a flat one have been presented in [12].

(2b) A third approach avoids the latter step of extending the results to physical backgrounds by computing instantly the flow equations for the fluctuation correlation functions for general backgrounds. This has been investigated in [18-21]. As in the other approaches, the background field approximation has been used for higher correlation functions. At present, this holds for all correlation functions beyond the one-point function of the fluctuation field.

\section{GENERAL FRAMEWORK}

In the present work we develop an approach in the class (2b). The present work does a qualitative step towards background independence and diffeomorphism invariance in asymptotically safe gravity by computing fluctuation correlation functions up to the three-point function as well as the full $f(R)$-potential of the background field. As already mentioned in the introduction, we compute the fixed point potential $f^{*}(R)$ for $k \rightarrow \infty$ but the present approach also allows for its computation in the physical limit $k \rightarrow 0$. This potential certainly has interesting applications in cosmology. The interplay of asymptotically safe gravity and cosmology is investigated in e.g. [75,94-116], and we hope to add to this in the near future.

The present approach is built on the vertex expansion setup to quantum gravity put forward in [3-8,10-14]. 
However, instead of expanding about the flat background, we consider for the first time coupling constants of the dynamical graviton field as arbitrary functions of the background curvature. We restrict ourselves to spherical backgrounds. A key point for this is the construction of an approximate momentum space, which allows us to utilize the previously developed techniques of running metric correlators in momentum space. With the resulting curvature-dependent dynamical couplings we find viable ultraviolet fixed point functions for all curvatures of the spherical background considered. Interestingly these fixed point functions of the effective couplings are almost curvature independent: the couplings try to counterbalance the explicit curvature dependence and thus try to keep the fixed point curvature independent. The fixed point functions provide further evidence in favour of the asymptotic safety scenario.

\section{A. FRG and Nielsen identities for gravity}

In order to compute correlation functions in quantum gravity we utilize the FRG approach to gravity [2]. In this approach the functional integral involves a momentum dependent mass function $R_{k}$, which acts as an infrared regulator suppressing momenta $p^{2} \lesssim k^{2}$ relative to the cutoff scale $k$. This leads to a scale-dependent effective action $\Gamma_{k}[\bar{g}, \phi]$, which includes contributions from high momentum fluctuations. Here the dynamical metric $g_{\mu \nu}=$ $\bar{g}_{\mu \nu}+\sqrt{Z_{h} G_{N}} h_{\mu \nu}$ is expanded around a nondynamical background metric $\bar{g}$ with the fluctuations $h$. The fluctuation field is rescaled with Newton's coupling such that it has the standard mass-dimension one of a bosonic field. In this work we utilize a linear metric split and we restrict $\bar{g}$ to spherical backgrounds. Combined with ghost fields $c, \bar{c}$ we denote the fluctuation superfield $\phi=(h, c, \bar{c})$. The scale-dependence of $\Gamma_{k}$ is then dictated by the flow equation [77-79],

$$
\partial_{t} \Gamma_{k}=\frac{1}{2} \operatorname{Tr}\left[G_{h h, k} \partial_{t} R_{h, k}\right]-\operatorname{Tr}\left[G_{\bar{c} c, k} \partial_{t} R_{c, k}\right],
$$

with the graviton and ghost regulators $R_{h, k}$ and $R_{c, k}$ respectively. The regulator terms are diagonal (symplectic) in field space, hence the diagonal graviton and (symplectic) ghost propagators, $G_{h h, k}$ and $G_{\bar{c} c, k}$, read

$$
G_{k}=\left(\Gamma_{k}^{(0,2)}+R_{k}\right)^{-1},
$$

with the general one-particle irreducible correlation functions given as derivatives of the effective action,

$$
\Gamma^{(n, m)}[\bar{g}, h]=\frac{\delta \Gamma[\bar{g}, h]}{\delta \bar{g}^{n} \delta h^{m}} .
$$

In (1) we have introduced the derivative with respect to the RG time $t=\log k / k_{\text {in }}$ where $k_{\text {in }}$ is a reference scale, usually taken to be the initial scale. The trace implies integrals over continuous and sums over discrete indices.

An important issue in quantum gravity is the background independence of physical observables. They are expectation values of diffeomorphism invariant operators, and hence do not depend on the gauge fixing. Examples for such observables are correlations of the curvature scalar. Another relevant example is the free energy of the theory, $-\log Z[\bar{g}, J=0]$, with $\delta Z[\bar{g}, J=0] / \delta \bar{g}=0$. These observables cannot depend on the choice of the background metric, which only enters via the gauge fixing. The latter fact is encoded in the NI for the effective action: The difference between background derivatives and fluctuation derivatives is proportional to derivatives of the gauge fixing sector,

$\mathrm{NI}=\frac{\delta \Gamma}{\delta \bar{g}_{\mu \nu}}-\frac{\delta \Gamma}{\delta h_{\mu \nu}}-\left\langle\left[\frac{\delta}{\delta \bar{g}_{\mu \nu}}-\frac{\delta}{\delta \hat{h}_{\mu \nu}}\right]\left(S_{\mathrm{gf}}+S_{\mathrm{gh}}\right)\right\rangle=0$,

where $S_{\mathrm{gf}}$ is the gauge fixing term and $S_{\mathrm{gh}}$ is the corresponding ghost term, and $h_{\mu \nu}=\left\langle\hat{h}_{\mu \nu}\right\rangle$. Note that (4) is nothing but the Dyson-Schwinger equation for the difference of derivatives with respect to $\bar{g}$ and $h$. For the fully diffeomorphism-invariant Vilkovisky-deWitt or geometrical effective action the relation (4) is even more concise: the split is not linear and we have $g=\bar{g}+f(\bar{g}, h)$, where $f(\bar{g}, h)=\sqrt{G_{N}} h+O\left(h^{2}\right)$ depends on the Vilkovisky connection. The NI then reads

$$
\mathrm{NI}_{\mathrm{geo}}=\frac{\delta \Gamma_{\mathrm{geo}}}{\delta \bar{g}_{\mu \nu}}-\mathcal{C}(\bar{g}, h) \frac{\delta \Gamma_{\mathrm{geo}}}{\delta h_{\mu \nu}}=0,
$$

where $\mathcal{C}(\bar{g}, h)$ is the expectation value of the (covariant) derivative of $h(\bar{g}, g)$, for a discussion in the present FRG setting see $[15,82-84]$.

The NIs, (4) and (5), entail that in both cases the effective action is not a function of $g=\bar{g}+h$ or $g=\bar{g}+f(\bar{g}, h)$ respectively. This property holds for general splits, and prevents the simple expansion of the effective action in terms of diffeomorphism invariants. Apart from this disappointing consequence of the NIs, it also entails good news: the effective action only depends on one field as background and fluctuation derivatives are connected.

An important property that follows from background independence is the fact that a solution of the background equation of motion (EoM)

$$
\left.\frac{\delta \Gamma[\bar{g}, h]}{\delta \bar{g}_{\mu \nu}}\right|_{\bar{g}=\bar{g}_{\mathrm{eom}}, h=0}=0,
$$

is also one of the quantum EoM,

$$
\left.\frac{\delta \Gamma[\bar{g}, h]}{\delta h_{\mu \nu}}\right|_{\bar{g}=\bar{g}_{\mathrm{eom}}, h=0}=0 .
$$


see e.g. [93] for a discussion of this in Yang-Mills theories. In (6) and (7) we have already taken the standard choice $h=0$ but the statement hold for general combinations $\bar{g}_{\mathrm{EoM}}(h)$ that solves either of the equations. The concise form (5) for the geometrical effective action makes it apparent that a solution of either EoM, (6) or (7), also entails a solution of the other one. Note that at $h=0$ we have $\mathcal{C}(\bar{g}, 0)=\mathbb{1}$.

Even though less apparent, the same holds true for the effective action in the linear split: to that end we solve the quantum EoM (7) as an equation for $\bar{g}_{\text {eom }}(h)$. As the current $J$ in the generating functional simply is $J=\delta \Gamma / \delta h$, the quantum EoM implies the vanishing of $J$ and the effective action is given by $\Gamma\left[\bar{g}_{\text {eom }}(0), 0\right]=-\log Z[\bar{g}, J=0]$, the free energy. However, we have already discussed that $\log Z[\bar{g}, 0]$ is background-independent and it follows that (6) holds.

The above properties and relations are a cornerstone of the background formalism as they encode background independence of observables. The NIs also link background diffeomorphism invariance to the Slavnov-Taylor identities (STIs) that hold for diffeomorphism transformations of the fluctuation field: the quantum deformation of classical diffeomorphism symmetry is either encoded in the expectation value of the gauge fixing sector or in the expectation value $\mathcal{C}(\bar{g}, h)$.

At finite $k$, the regulator term introduces a genuine dependence on the background field. Then $\log Z_{k}[\bar{g}, 0]$ is not background independent. Consequently the STIs turns into modified STIs (mSTIs) and the NIs turn into modified NIs (mNIs). For the linear split, the mNI reads

$$
\mathrm{mNI}=\mathrm{NI}-\frac{1}{2} \operatorname{Tr}\left[\frac{1}{\sqrt{\bar{g}}} \frac{\delta \sqrt{\bar{g}} R_{k}[\bar{g}]}{\delta \bar{g}_{\mu \nu}} G_{k}\right]=0,
$$

see [80,81] for details and [117] for an application to quantum gravity. Importantly the right-hand side of (8) signals the loss of background independence. It is proportional to the regulator and vanishes for $k \rightarrow 0$ where background independence is restored. A similar violation of background independence linear in the regulator is present in the geometrical approach, see [15,82-84].

In summary this leaves us with nonequivalent solutions to the EoMs in the presence of the regulator: a solution of the quantum EoM (7) does not solve the background EoM (6). However, typically the asymptotically safe UV regime of quantum gravity is accessed in the limit $k \rightarrow \infty$ as this already encodes the important scaling information in this regime. In the present paper we also follow this strategy and hence we have to deal with different solutions of background and quantum EoMs, if they exist at all. Note that the right-hand side of the $\mathrm{mNI}$ is simply the expectation value of the background derivative of the regulator term. Accordingly it is the background EoM that is deformed directly by the presence of the regulator while the quantum
EoM feels its influence only indirectly. Therefore it is suggestive to estimate the physical UV-limit of the EoM in the limit $k \rightarrow 0$ by the quantum EoM in the limit $k \rightarrow \infty$.

Studies in asymptotically safe quantum gravity have focused so far on finding solutions to (6). For instance in [116] they did not find a solution to (6) in a polynomial expansion with the background field approximation. Other approaches with the background field approximation found a solution with the exponential parametrization [118,119] and within the geometrical approach $[43,49]$. In this work we are for the first time able to disentangle (6) and (7) in a quantum gravity setting and look for separate solutions to the EoMs.

We disentangle the background and fluctuation field by expanding the scale dependent effective action around a background according to

$$
\Gamma_{k}[\bar{g}, h]=\sum_{n=0}^{\infty} \frac{1}{n !} \Gamma_{k}^{(0, n)}[\bar{g}, h=0] h^{n} .
$$

The flow equations that govern the scale-dependence of the vertex functions are obtained by $n$ field derivatives of the flow equation for the effective action (1). They are depicted in a diagrammatic language in Fig. 1 for cases $n=2$ and $n=3$. These flow equations are familiar from computations on a flat background [3,5,6,11], here however all propagators and vertices depend nontrivially on the background.

From here on we drop the index $k$ to improve readability, the scale dependence of the couplings, correlation functions and wave function renormalizations is implicitly understood.

\section{B. Background independence in nonperturbative expansion schemes}

It is important to discuss the relations of the approaches described in Sec. II in particular for future developments and the full resolution of physical background independence. This chapter extends a similar discussion from [11] in the context of modified STIs for diffeomorphism

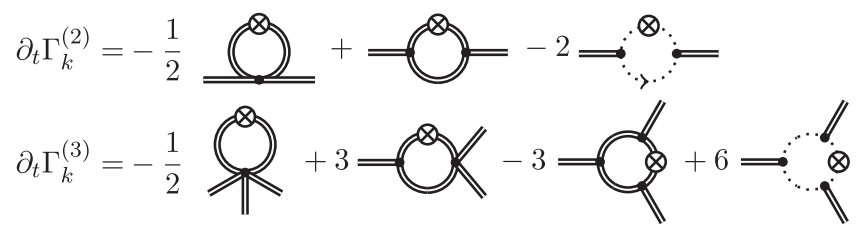

FIG. 1. Displayed are the diagrammatic representations of the flows of the graviton two- and three-point functions. Double and dashed lines represent dressed graviton and ghost propagators respectively, while filled circles denote dressed vertices. Crossed circles stand for regulator insertions. All quantities are explicit background curvature dependent and carry further background curvature dependence via the spectral value of the respective vertex/propagator. 
transformations to NIs. Despite its importance one may skip this chapter for a first reading as its results are not necessary for the derivations and computations presented in this work.

We have technically very different options to access physical background independence of quantum gravity. Seemingly they have different advantages and disadvantages. For example, approach (1) via the NIs has the charm of directly implementing background independence. In turn, the results of (2b) may apparently not satisfy the NIs.

For resolving this issue it is instructive to discuss approach (2a). There the fluctuation correlation functions are computed for a specific background. Results for general backgrounds have then to be obtained with an expansion/ extension of the results for the specific background. This could be done via the NIs in which case background independence is guaranteed. This procedure for guaranteeing STIs and NIs has been discussed in detail in [120] in the context of non-Abelian gauge theories, and in [11] for gravity. We briefly repeat and extend the structural argument presented there: First we notice that the functional equations for all correlation functions can be cast in the form

$$
\Gamma^{(n, m)}[\bar{g}, h]=\mathrm{FRG}_{n, m}\left[\left\{\Gamma^{(i \leq n, 2 \leq j \leq m+2)}[\bar{g}, h]\right\}, \bar{g}\right] .
$$

Equation (10) follows from integrating the functional renormalization group equations for $\Gamma^{(n, m)}$, which have precisely the same structure for all theories: the flows of $\Gamma^{(n, m)}$ are given by one-loop diagrams with full propagators and full vertices. The latter are given in terms of the correlation functions $\left\{\Gamma^{(i \leq n, 2 \leq j \leq m+2)}\right\}$, see e.g. $[83,120]$. This also entails that the lowest fluctuation correlation function that contributes to the diagrams is the two-point function, i.e. the propagator.

In gravity (10) follows straightforwardly from (1) by integrating the flow equation and taking $\bar{g}$ - and $h$-derivatives. As a side remark we note that the order of derivatives on the right-hand side is different within other functional approaches. For example, for Dyson-Schwinger equations (DSE) the right-hand side $\mathrm{DSE}_{n, m}$ for the $\Gamma^{(n, m)}$ depends on $\left\{\Gamma^{(i \leq n, j \leq m+r-2)}\right\}$ and contain up to $r-2$-loop diagrams. Here $r$ is the highest order of the field in the classical action, see e.g. [83]. In typical examples of renormalizable theories we have $r=3,4$, but in gravity we have $r=\infty$. This singles out the flow equation for gravity as the only functional approach that only connects a finite order of correlation functions in each equation. The coupling of the whole tower of equations then comes from the highest order correlation functions on the right-hand side. In turn, each DSE already contains all orders on the right-hand side of (10), that is $2 \leq j$ without upper bound. Similar statements as for the DSE hold for 2PI or $n$ PI hierarchies.

Importantly, for all functional approaches the right-hand side of (10) goes only up to the same order of background metric derivatives, $i \leq n$. This allows us to view (10) as functional relations for the highest order background metric correlation functions that have as an input $\left\{\Gamma^{(n-1, m)}\right\}$. Moreover, the NI relates a derivative with respect to $\bar{g}$ to one with respect to $h$. For emphasizing the similarities to the functional relations (10) we rewrite the NI. For simplicity we use the linear split NI, (4) and (8),

$$
\begin{aligned}
\Gamma^{(n, m)}[\bar{g}, h]= & \Gamma^{(n-1, m+1)}[\bar{g}, h] \\
& +\mathcal{N}_{n, m}\left[G,\left\{\Gamma^{(i \leq n-1, j \leq m+1)}[\bar{g}, h]\right\}, \bar{g}\right],
\end{aligned}
$$

where $\mathcal{N}$ stands for the expectation value in (4), and additionally for the regulator loop in (8), and we have singled out the propagator $G$ for elucidating the orders of the correlation functions on both sides. Importantly, (11) makes the fact apparent that for the NI, (4) and (8), the order of background derivatives is at most $n-1$. Note also that (11) is nothing but the difference of the DysonSchwinger equation for $h$ and $\bar{g}$ derivatives. In this difference the terms with the higher vertices with $j \geq m+2$ drop out.

In summary this leaves us with two towers of functional relations. While the first one, (10) describes the full set of correlation functions, the second one, (11) can be used to iteratively solve the tower of mixed fluctuation-background correlations on the basis of the fluctuating correlation functions $\left\{\Gamma^{(0, m)}\right\}$. In both cases we can solve the system for the higher-order correlations of the background on the basis of the lower order correlations. If we use (11) with an iteration starting with the results from the flow equation for $\left\{\Gamma^{(0, m)}\left[\bar{g}_{\mathrm{sp}}, h\right]\right\}$ for a specific background $\bar{g}_{\mathrm{sp}}$, this closure of the system automatically satisfies the NI. Accordingly, any set of fluctuation correlation functions $\left\{\Gamma^{(0, m)}\left[\bar{g}_{\mathrm{sp}}, h\right]\right\}$ can be iteratively extended to a full set of fluctuationbackground correlation functions in an iterative procedure. Note that this procedure can be also applied to the case (2b).

While this seems to indicate that satisfying the symmetry identities is not relevant (it can be done for all inputs), it points at a more intricate structure already known from non-Abelian gauge theories. To that end let us assume we have derived a global unique solution of all correlation functions within this iterative procedure starting from the fluctuations correlation functions. If no approximation is involved, this solution automatically would satisfy the full set of functional relations for $\left\{\Gamma^{(n, m)}\right\}$ that can be derived from the flow equation. However, in the presence of approximations these additional functional relations represent infinite many additional constraints on the iterative solution. These constraints are bound to fail in generic nonperturbative approximation schemes as any functional relation triggers specific resummations in given approximations. It is a priori not clear which of the functional relations are more important. Note also that typically the 
iterative solutions of the symmetry identities are bound to violate the locality constraints of local quantum field theories that are tightly connected to the unitarity of the theory. In conclusion it is fair to say that only a combination of all approaches is likely to provide a final resolution of physical background independence and diffeomorphism invariance in combination with unitarity.

\section{VERTICES IN CURVED BACKGROUNDS}

This section contains technical details about the construction of an approximate momentum space and the vertex flow equations on curved backgrounds. If one is not interested in these details, one may proceed to Sec. V.

\section{A. Spectral decomposition}

We extend our previous expansion schemes about the flat Euclidean background to one that allows for arbitrary constant curvatures. To that end we first discuss the procedure at the example of the propagators: propagators for nontrivial metrics $\bar{g}$ with constant curvature can be written in terms of the scalar Laplacian $\Delta_{\bar{g}}=-\bar{\nabla}^{2}$ and curvature terms proportional to the background scalar curvature $\bar{R}$,

$$
G=G\left(\Delta_{\bar{g}}, \bar{R}\right) .
$$

For the flat metric (12) reduces to $G\left(p^{2}, 0\right)$, where $p^{2}$ are the continuous spectral values of the flat scalar Laplacian. In a spectral basis the propagator is diagonal and reads for general curvatures

$$
\left.\left\langle\varphi_{\lambda}|G| \varphi_{\lambda}\right\rangle\right|_{\lambda=p^{2}}=G\left(p^{2}, \bar{R}\right),
$$

and $\lambda=p^{2}$ are the discrete or continuous eigenvalues for the given metric, and $\left\{\left|\varphi_{\lambda=p^{2}}\right\rangle\right\}$ is the orthonormal complete basis of eigenfunctions of the scalar Laplacian

$$
\Delta_{\bar{g}}\left|\varphi_{\lambda}\right\rangle=\lambda\left|\varphi_{\lambda=p^{2}}\right\rangle
$$

see Appendix A for explicit expression for the propagator. The tricky part in this representation are the vertices, which are operators that map $n$ vectors onto the real numbers. For example the three-point function can be written in a spectral representation in terms of an expansion in the tensor basis with eigenfunctions of $\Delta_{\bar{g}}$,

$\Gamma^{(3)}=\underbrace{}_{\lambda_{1}, \lambda_{2}, \lambda_{3}} \Gamma^{(3)}\left(\lambda_{1}, \lambda_{2}, \lambda_{3}, \bar{R}\right)\left\langle\varphi_{\lambda_{1}}\right| \otimes\left\langle\varphi_{\lambda_{2}}\right| \otimes\left\langle\varphi_{\lambda_{3}}\right|$,

where the spectral values in general also depend on the curvature and $\mathbb{E}$ runs over discrete or continuous spectral values. Also, $\mathbb{E}$ may also include a nontrivial spectral measure weight $\mu(\lambda)$. The representation of the higher $n$-point functions follows straightforwardly from (15). Inserting this into the flow equation of the inverse propagator, we arrive at

$$
\begin{aligned}
\partial_{t} \Gamma^{(2)}(\lambda, \bar{R})= & -\frac{1}{2} \bigcup_{\lambda_{1}} \Gamma^{(4)}\left(\lambda, \lambda, \lambda_{1}, \lambda_{1}, \bar{R}\right)\left(G \dot{R}_{k} G\right)\left(\lambda_{1}, \bar{R}\right) \\
& +\sum_{\lambda_{2}, \lambda_{3}} \Gamma^{(3)}\left(\lambda, \lambda_{2}, \lambda_{3}, \bar{R}\right) G\left(\lambda_{2}, \bar{R}\right) \\
& \times\left(G \dot{R}_{k} G\right)\left(\lambda_{3}, \bar{R}\right) \Gamma^{(3)}\left(\lambda_{3}, \lambda_{2}, \lambda, \bar{R}\right),
\end{aligned}
$$

where we denoted $\dot{R}_{k}=\partial_{t} R_{k}$. The vertex functions $\Gamma^{(n)}$ are complicated functions of $\lambda_{i}$.

On a flat background, the eigenfunctions of the Laplace operator are also eigenfunctions of the partial derivatives and the representation of the vertex functions follows trivially. On a curved background, however, the covariant derivatives do not commute with the Laplace operator and the representation of uncontracted covariant derivatives on the set of functions $\left\{\left|\varphi_{\lambda=p^{2}}\right\rangle\right\}$ is complicated. One could tackle this problem with e.g. off-diagonal heat-kernel methods, but then a derivative expansion in momenta and curvature is necessary [12].

In this work we construct an approximate momentum space on a curved background, which facilitates computations considerably and allows for full momentum and curvature dependences. In order to derive the vertex functions, we first take functional derivatives with respect to the Einstein-Hilbert action on an arbitrary background. The result is a function depending on the Laplacian, products of covariant derivatives with respect to coinciding or different spacetime points and explicit curvature terms. In the expression for the vertex functions we symmetrize all covariant derivatives, which produces further $\bar{R}$-terms

$$
\bar{\nabla}^{\mu} \bar{\nabla}^{\nu}=\frac{1}{2}\left\{\bar{\nabla}^{\mu}, \bar{\nabla}^{\nu}\right\}+\bar{R} \text {-terms. }
$$

In the curved momentum space approximation here, the product of symmetrized covariant derivatives acts on the set $\left\{\left|\varphi_{\lambda=p^{2}}\right\rangle\right\}$ according to

$\bar{\nabla}_{1} \cdot \bar{\nabla}_{2}=p_{g} \cdot q_{g}=\sqrt{p^{2}} \sqrt{q^{2}} x$, with $\quad x=\cos \theta_{\text {flat }}$,

with an integration measure $\int \sqrt{1-\sin ^{2} \theta} \mathrm{d} \cos \theta$. The integration measure is chosen such that in the limit $\bar{R} \rightarrow 0$ precisely the flat results are obtained. As a consequence, in this approximation $\mathbb{E}$ factorizes into an angular integration and a sum/integration over the spectral values of $\Delta_{\bar{g}}$. According to (18), external spectral values are described by the angle to the internal one and their absolute 
values, which appear as parameters that can be treated as real numbers. We emphasize that this curved momentum space approximation has the correct flat background limit by construction and is correct for all terms that contain only Laplace operators. A comparison of the approximation as a function of the background curvature is detailed in Appendix C. With the above approximation associated with covariant derivatives, we arrive at a relatively simple flat-background-type representation of the flow equation in terms of angular integrals and spectral values $p_{i}^{2}=\lambda_{i}$

$$
\begin{aligned}
\partial_{t} \Gamma^{(2)} & (\lambda, \bar{R}) \\
= & -\frac{1}{2} y_{\lambda_{1}} \int \mathrm{d} \Omega \Gamma^{(4)}\left(\lambda, \lambda_{1}, x, \bar{R}\right)\left(G \dot{R}_{k} G\right)\left(\lambda_{1}, \bar{R}\right) \\
& +y_{\lambda_{1}} \int \mathrm{d} \Omega \Gamma^{(3)}\left(\lambda, \lambda_{1}, x, \bar{R}\right) G\left(\lambda_{1}+\lambda+\sqrt{\lambda \lambda_{1}} x, \bar{R}\right) \\
& \times\left(G \dot{R}_{k} G\right)\left(\lambda_{1}, \bar{R}\right) \Gamma^{(3)}\left(\lambda, \lambda_{1}, x, \bar{R}\right) .
\end{aligned}
$$

The total $\bar{R}$-dependence of the flow equation enters via the explicit $\bar{R}$-terms in the vertex functions, the symmetrized covariant derivatives and the spectral values. The generalization to flows of higher-order vertex functions is straightforward.

\section{B. Vertex construction}

The basic ingredients in the flow equations in Fig. 1 are the vertex functions $\Gamma^{(n)}$. We build on the parametrization for vertex functions introduced in $[5,6,11,121]$. In contrast to earlier truncations with vertex expansions around a flat background, all quantities exhibit explicit $\bar{R}$-dependence. Hence, our general ansatz is given by

$$
\Gamma^{\left(\phi_{1} \ldots \phi_{n}\right)}(\mathbf{p}, \bar{R})=S_{\mathrm{EH}}^{\left(\phi_{1} \ldots \phi_{n}\right)}\left(\mathbf{p} ; G_{n}(\bar{R}), \Lambda_{n}(\bar{R}), \bar{R}\right),
$$

where $\mathbf{p}=\left(p_{1}, \ldots, p_{n}\right)$ is the collection of spectral values of the external legs and $S_{\mathrm{EH}}$ is the gauge-fixed EinsteinHilbert action

$$
S_{\mathrm{EH}}=\frac{1}{16 \pi G_{N}} \int \mathrm{d}^{4} x \sqrt{g}(2 \Lambda-R)+S_{\mathrm{gf}}+S_{\mathrm{gh}} .
$$

We employ a De-Donder-type linear gauge condition in the Landau limit, $\alpha=\beta=0$.

In (20) the Newton's constant and the cosmological constant of the classical gauge fixed Einstein Hilbert action are getting replaced with $G_{n}(\bar{R})$ and $\Lambda_{n}(\bar{R})$, respectively. They parametrize the gravitational coupling and the momentum-independent part of the $n$-point function. Note that the graviton $n$-point function in (20) is proportional to $G_{n}^{n / 2-1}$ as well as to $Z_{h}^{n / 2}$ due to the rescaling of the graviton fluctuation field to a field with mass dimension one. This is captured with the split $g_{\mu \nu}=\bar{g}_{\mu \nu}+\sqrt{Z_{h} G_{N}} h_{\mu \nu}$.
The wave function renormalization is in general momentum and background-curvature dependent, $Z_{h}=Z_{h}\left(p^{2}, \bar{R}\right)$.

The propagator is a pure function of $\Delta_{\bar{g}}$ and $\bar{R}$, while the vertices with $n>2$ are functions of $\Delta_{\bar{g}}, \bar{\nabla}_{\mu}, \bar{R}, \bar{R}_{\mu \nu}$ and $\bar{R}_{\mu \nu \rho \sigma}$. Restricting ourselves to a background sphere, the dependence on the Ricci- and the Riemann-tensor reduces to a dependence on the constant background curvature $\bar{R}$. With the approximation constructed in the last section, we deal with the covariant derivatives $\bar{\nabla}_{\mu}$ in the vertices. We set the anomalous dimensions

$$
\eta_{\phi_{i}}\left(p^{2}, \bar{R}\right):=-\partial_{t} \ln Z_{\phi_{i}}\left(p^{2}, \bar{R}\right),
$$

throughout this work equal to zero. In the flat computation $[6,11]$ this approximation led to qualitatively reasonable results. The graviton three-point function is evaluated at the point of symmetric spectral values,

$$
p:=\left|p_{1}\right|=\left|p_{2}\right|, \quad \theta_{\text {flat }}=2 \pi / 3 .
$$

We also introduce the dimensionless variables

$r:=\bar{R} k^{-2}, \quad g:=G k^{2}, \quad \mu:=-2 \Lambda_{2} k^{-2}, \quad \lambda_{3}:=\Lambda_{3} k^{-2}$.

From the graviton two-point function we extract the massparameter $\mu(r)$, while from the graviton three-point function we extract the gravitational coupling $g(r)$ and the coupling of its momentum independent part $\lambda_{3}(r)$. In Appendix B we give a derivation and display the flow equations. In summary the set of couplings in the present truncation is given by

$$
\left(g(r), \mu(r), \lambda_{3}(r)\right) .
$$

\section{Flow equations and trace evaluation}

With the construction presented in the last sections, we are left with an explicit expression for the flow of the twoand the three-point function. The flow of the two-point is of the form (19) and the three-point function has a similar form according to the diagrammatic representation in Fig. 1. After projection the resulting flow equations take the form (B5) and (B6). In this work we are interested in the fixed point equations, which are differential equations with respect to $r$ due to the dependence on the background curvature. According to the factorization property of the approximate curved momentum space construction, we evaluate the angular integration in a straightforward manner in complete analogy to a flat background computation. We are then left with the evaluation of traces of the form

$$
y_{\lambda} f(\lambda, r),
$$


for functions of the curvature $r$ and the spectral value $\lambda$ as well as the couplings. In order to include the effects of the background curvature we perform a spectral sum over a four-sphere. On a four-sphere the spectrum of the scalar Laplacian is given by

$$
\omega(\ell)=\frac{\ell(3+\ell)}{12} r,
$$

with multiplicities

$$
m=\frac{(2 \ell+3)(\ell+2) !}{6 \ell !},
$$

with $\ell$ taking integer values $\ell \geq 0$. Since we are left with only scalar spectral values we replace the spectral values by

$$
\lambda \rightarrow \omega(\ell)
$$

and replace

$$
y_{\lambda} \rightarrow V^{-1} \sum_{\ell=2}^{\ell_{\max }} m(\ell),
$$

where the exact sum is achieved for $\ell_{\max }=\infty$ and we divide by the volume of a four sphere $V=\frac{384 \pi^{2}}{k^{4} r^{2}}$. Note, that we exclude the zero modes and start the spectral sum at $\ell=2$. This does not affect the result for small curvature $r$. Performing the spectral sums one then obtains the traces. However, in most cases a closed form for the sums cannot be obtained and we have to resort to cutting the spectral sums off at a finite value $\ell_{\max }$. Nonetheless since each trace involves a regulator function that cuts modes off at order $\omega(\ell) \approx k^{2}$ for nonzero $r$ the spectral sum is only sensitive to the modes $\omega(\ell)<k^{2}$, which are finite in number. However, in the limit of vanishing curvature the spectral sum needs to be extended to infinite order, as all modes are only regulator suppressed for large $r$ according to $\exp \left(-\lambda_{n} r\right)$, but become important once $r \approx 1 / \lambda_{n}$. In fact, we need the limit $r \rightarrow 0$ in order to set the boundary conditions of the fixed-point differential equations. It is obvious that there is only one physical initial conditions that fixes the solution of the fixed point differential equation uniquely, and that is the initial condition obtained from the flat background limit. In fact, a proper initial condition is also necessary from a mathematical point of view if one requires a finite derivative, $g^{\prime}(r)<\infty$. One infers from (B5) and (B6), that the derivative of $g(r)$ diverges in the limit $r \rightarrow 0$ if the initial conditions are not chosen appropriately. However, as argued above, this limit cannot be calculated in practice with spectral sums as all modes contribute. In the smallcurvature region the trace is evaluated by the early-time heat-kernel expansion where the leading order gives the flat-background momentum integrals. In this case we write the Laplace transform

$$
\searrow_{\lambda} f(\lambda, r)=\frac{1}{V} \int_{0}^{\infty} \mathrm{d} s \operatorname{Tr}\left[e^{-s \Delta_{g}}\right] \tilde{f}(s, r),
$$

and one expands the trace of the heat kernel in the scalar curvature $r$ and the explicit dependence on $r$ coming from $\tilde{f}(s, r)$. For small curvature the early-time heat-kernel expansion is given by

$\frac{1}{V} \int_{0}^{\infty} \mathrm{d} s \operatorname{Tr}\left[e^{-s \Delta_{g}}\right] \tilde{f}(s, r)=\frac{1}{(4 \pi)^{2}}\left(Q_{2}[f]+Q_{1}[f] \frac{r}{6}+\cdots\right)$,

where for $n>0$

$$
Q_{n}[f]=\frac{1}{\Gamma(n)} \int \mathrm{d} \lambda \lambda^{n-1} f(\lambda, r)
$$

Using this heat-kernel expansion we translate the physical initial condition to finite $r$ where we connect to the spectral sum. In particular we determine the curvature-dependent couplings as polynomials in the curvature $r$. The heat kernel provides the asymptotic limit $r \rightarrow 0$ which can be reproduced by the spectral sum in the limit $\ell_{\max } \rightarrow \infty$. Thus, while the spectral sum with finite $\ell_{\max }$ captures the large $r$ behavior of the trace, the heat kernel expanded to a finite order in $r$ captures the small $r$ behavior. Both connect smoothly for finite but small $r$, for details see Appendix D.

\section{RESULTS}

In this section we present the results of the given setup. First, we discuss the fixed point solutions of the beta functions related to the fluctuation field couplings. In our approach with curvature-dependent couplings, these solutions are fixed point functions. Subsequently, we analyse the background effective potential, which is calculated on the solution of the fluctuation field fixed point solution, with and without standard model matter content. Last we look for solution of the quantum EoM and compare to solutions of the background EoM.

\section{A. Fixed point solutions}

The beta functions for a coupling $g_{i}(r)$ in the present framework are partial differential equations. Schematically, the equation a coupling $g_{i}$ takes the form

$\partial_{t} g_{i}(r)=g_{i}(r) A\left(g_{j}, \eta_{h}\right)+2 r g_{i}^{\prime}(r)+$ Flow $_{g_{i}}\left(g_{j}, r\right)$,

with a coefficient $A$ that depends on the other scale-depend parameters $g_{j}$. For explicit expressions we refer to Appendix B. The fixed point equations are then obtained by setting $\partial_{t} g_{i}(r) \equiv 0$ and we are left with a system of ordinary differential equations. The initial condition is imposed at $r=0$ and is chosen such that it matches the computation in a flat background [6]. For details see 
Sec. IV C. The UV fixed point values for a flat background, $g_{i}(r)=g_{i, 0}$, are given by

$$
\left(g_{0}^{*}, \lambda_{3,0}^{*}, \mu_{0}^{*}\right)=(0.60,-0.12,-0.38) .
$$

with the critical exponents $\theta$, which are the negative eigenvalues of the stability matrix,

$$
\left(\theta_{i, 0}\right)=(-3.5,2.1 \pm 2.1 i)
$$

These values differ slightly from the ones in [6] since we use the gauge parameter $\beta=0$ and the exponential regulator, see (A5). Taking this difference into account, the agreement is remarkable and highlights the insensitivity of our results with respect to the gauge and the regulator.

In order to display our results, it is convenient and meaningful to introduce effective couplings that include the explicit $r$ dependence in the respective graviton $n$-point functions. According to (A6) and (B6), these are given by

$$
\begin{aligned}
g_{\mathrm{eff}}(r) & =g(r), \\
\mu_{\mathrm{eff}}(r) & =\mu(r)+\frac{2}{3} r, \\
\lambda_{3, \mathrm{eff}}(r) & =\lambda_{3}(r)+\frac{1}{6} r .
\end{aligned}
$$

The interpretation and relevance of these effective couplings can be inferred for instance from the graviton twopoint function. In terms of $\mu_{\mathrm{eff}}(r)$, the transverse-traceless part of the graviton two-point function reads

$$
\Gamma^{(0,2)}=\left(\Delta+\mu_{\mathrm{eff}}(r)\right)
$$

i.e. it comprises the nonkinetic part of the correlator. As an aside, we mention that one could define $\lambda_{3 \text {,eff }}$ alternatively via (B5). The difference between the effective coupling for $\lambda_{3}$ in (B6) and (B5) arises from the different tensor projections. We choose to define $\lambda_{3, \text { eff }}$ via (B6) since the flow equation for $g$ is more important in this system.

The full, $r$-dependent fixed point solutions $\left(g^{*}(r)\right.$, $\left.\mu_{\mathrm{eff}}^{*}(r), \lambda_{3, \mathrm{eff}}^{*}(r)\right)$ are displayed in Fig. 2. We find a fixed point solution with all desired properties. First of all, the fixed point solution is characterized by a positive gravitational coupling $g(r)>0$, which decreases towards larger background curvatures. In order to get a feeling for the physical meaning of this behaviour, we consider the quantity $G(R) R=g(r) r$, i.e. the dimensionful Newton's coupling times the curvature. As this product is dimensionless, it can in principle be used to define an observable. In particular, we expect that this quantity is finite at the fixed point, which implies $g^{*}(r) \sim 1 / r$. One might interpret our fixed solution $g^{*}(r)$ as an onset of such a behavior. The solutions for the mass-parameter $\mu_{\text {eff }}(r)$ and $\lambda_{3, \text { eff }}(r)$ are almost curvature independent, which implies that the implicit curvature dependence cancels with the explicit one.

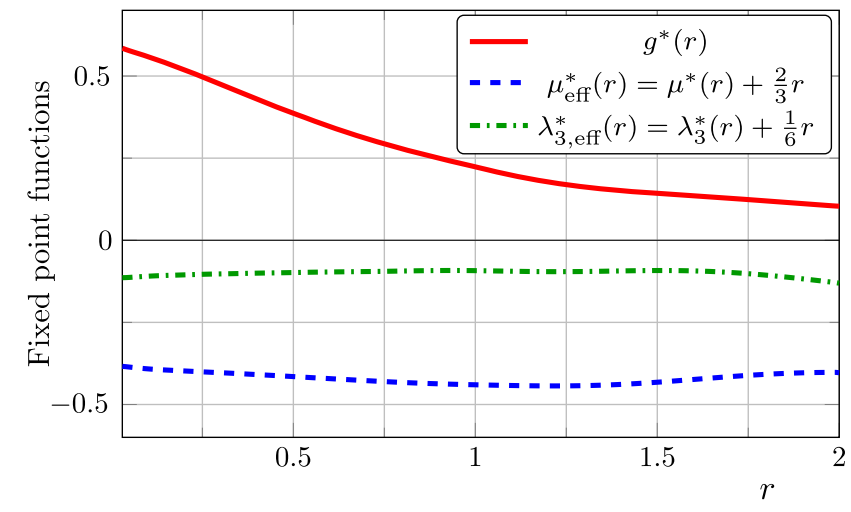

FIG. 2. Fixed point function solution for the system $\left(g^{*}(r)\right.$, $\left.\mu_{\mathrm{eff}}^{*}(r), \lambda_{3, \text { eff }}^{*}(r)\right)$ with the boundary condition from the first-order heat kernel. The solutions are stable in the whole investigated region. Note, that the effective couplings according to (37) are displayed.

Consequently, the behaviour is not so different from the one of the computation on a flat background.

The full solution shown in Fig. 2 can be expanded in powers of the dimensionless curvature, $g_{i}^{*}(r)=g_{i, 0}^{*}+$ $g_{i, 1}^{*} r+\mathcal{O}\left(r^{2}\right)$. The zeroth order is displayed in (35) and to linear order in $r$ we find

$$
\left(g_{1}^{*}, \lambda_{3,1}^{*}, \mu_{1}^{*}\right)=(-0.44,-0.09,-0.79) .
$$

with the critical exponents $\theta$ given by

$$
\left(\theta_{i, 1}\right)=(-5.7,0.27 \pm 3.1 i)
$$

We find two further UV attractive directions in the linear order of the background curvature. Further attractive directions of the UV fixed point that are linear in the background curvature were also found in [12].

\section{B. Background potential}

In the previous section we have presented the fixed point solution for the fluctuation field couplings. All background quantities depend on these dynamical couplings and have to be evaluated on the above solution. Along these lines we calculate a background field potential at the fixed point. The flow of the background potential is completely determined by the dynamical couplings of the two-point function. In particular, the background flow equation reads

$$
\partial_{t} \Gamma[\bar{g}, 0]=\frac{1}{2} \operatorname{Tr}\left[G \partial_{t} R_{k}\right]_{h h}-\left.\operatorname{Tr}\left[G \partial_{t} R_{k}\right]_{\bar{c} c}\right|_{\phi=0} .
$$

On a sphere, the background effective action is given by

$$
\Gamma[\bar{g}, 0]=\int \mathrm{d}^{4} x \sqrt{\bar{g}} k^{4} f\left(\bar{R} / k^{2}\right)=\frac{384 \pi^{2}}{r^{2}} f(r) .
$$


Denoting the right-hand side of (41) by $\mathcal{F}(r, \mu(r))$ we obtain a flow equation for the function $f(r)$ given by

$$
\frac{384 \pi^{2}}{r^{2}}\left(\partial_{t} f+4 f(r)-2 r f^{\prime}(r)\right)=\mathcal{F}(r, \mu(r))
$$

If we then look at the fixed point for $f^{*}(r)$ we find

$$
\frac{384 \pi^{2}}{r^{2}}\left(4 f^{*}(r)-2 r f^{* \prime}(r)\right)=\mathcal{F}\left(r, \mu^{*}(r)\right) .
$$

One then notes that the left-hand side is just the background EoM for $f(r)$-gravity on a constant curvature background. Thus when the function $\mathcal{F}\left(r, \mu^{*}(r)\right)$ vanishes we have a solution to the background EoM at the fixed point given by

$$
\mathcal{F}\left(r_{0}, \mu^{*}\left(r_{0}\right)\right)=0
$$

Equivalently we can look for a minimum of the function $f(r) / r^{2}$. In Fig. 3 we plot the background potential $f(r) / r^{2}$ for our full solution (left panel) as well as in comparison with other approximations (right panel). There we use $\mu_{\mathrm{eff}}^{*}(r)=\mu_{0}^{*}$ and $\mu^{*}(r)=\mu_{0}^{*}$ as given in (35). The first is seen to be a good approximation from Fig. 2 while the latter reduces our computation to an Einstein-Hilbert approximation. We observe that in the full solution and in the $\mu_{\mathrm{eff}}^{*}(r)=\mu_{0}^{*}$ approximation there are no solutions to the background EoM within the investigated curvature regime. This absence of a constant curvature solution is in agreement with studies of $f(R)$ gravity in the background field approximation [116], although solutions have been found in calculations exploiting the exponential parametrization $[118,119]$ and within the geometrical approach [43,49]. For the approximation $\mu^{*}(r)=\mu_{0}^{*}$, which corresponds to a pure Einstein-Hilbert computation, we find a minimum at

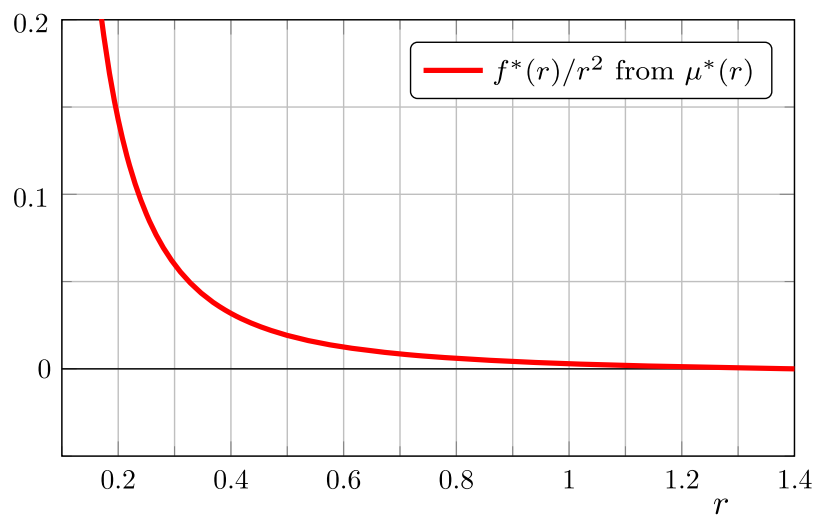

$r_{0}=0.97$. This is again in agreement with computations in the background field approximation $[122,123]$.

In a polynomial expansion around $r=0$ the background potential of the full solution would take the form

$$
f(r)=0.0065-0.0065 r+\mathcal{O}\left(r^{2}\right),
$$

and consequently we obtain fixed point values of the background Newton's coupling and the background cosmological constant according to

$$
\bar{g}^{*}=3.0, \quad \bar{\lambda}^{*}=0.50 .
$$

Note that $\bar{\lambda}=\frac{1}{2}$ is not a pole in our computation: the pole is only present in the graviton mass parameter $\mu(r)$. Surprisingly the fixed point value of $\bar{g}^{*}$ is rather large. We compare these values with the pure Einstein-Hilbert approximation, see blue dashed line in Fig. 3. We find

$$
f_{\mathrm{EH}}(r)=0.0065-0.021 r+\mathcal{O}\left(r^{2}\right),
$$

and consequently

$$
\bar{g}_{\mathrm{EH}}^{*}=0.94, \quad \bar{\lambda}_{\mathrm{EH}}^{*}=0.15 .
$$

These values are comparable to standard Einstein-Hilbert computations in the background field approximation as well as in fluctuation computations. Thus the large values in (47) are indeed triggered by the nontrivial $r$ dependence of the couplings.

We investigate the stability of the present results by treating $\mu_{\mathrm{eff}}^{*}(r)$ as a free parameter without curvature dependence. In this case, $\mu_{\text {eff }}^{*}(r)=\mu_{0}^{*}$ is a good approximation for our best solution as discussed above. By varying this parameter we see for which values a solution to the background EoM exists. With reference to Fig. 4 we find

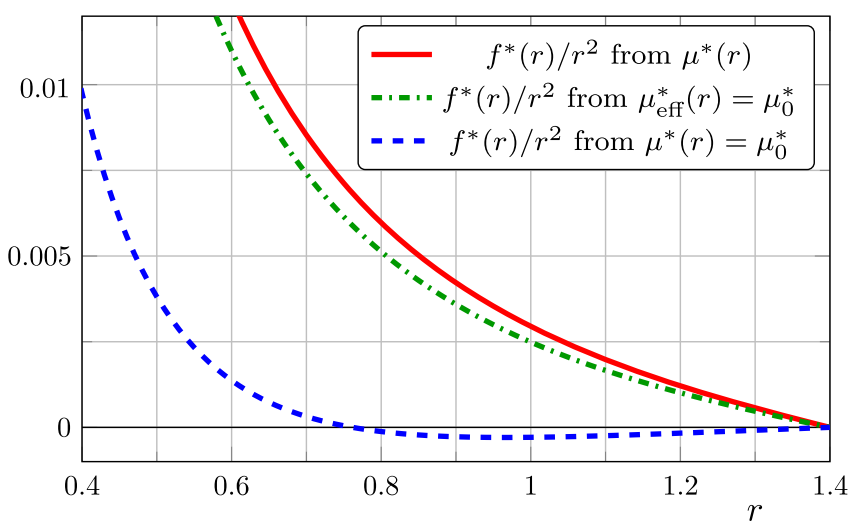

FIG. 3. Displayed are background potentials $f^{*}(r) / r^{2}$ obtained from the fixed point solution $\mu^{*}(r)$ (left and right panel) and from the approximations $\mu_{\mathrm{eff}}^{*}(r)=\mu_{0}^{*}$ and $\mu^{*}(r)=\mu_{0}^{*}$ (right panel). All curves are obtained with the condition $f^{*}(r=1.4)=0$. Other conditions just shift the potential $f^{*}(r) / r^{2}$ by a constant. The full solution does not contain a minimum, it becomes asymptotically flat. The approximation $\mu_{\mathrm{eff}}^{*}(r)=\mu_{0}^{*}$ is qualitatively very good, see also Fig. 2. The approximation $\mu^{*}(r)=\mu_{0}^{*}$ corresponds to a pure EinsteinHilbert computation. Here we find a minimum at $r_{0}=0.97$. 

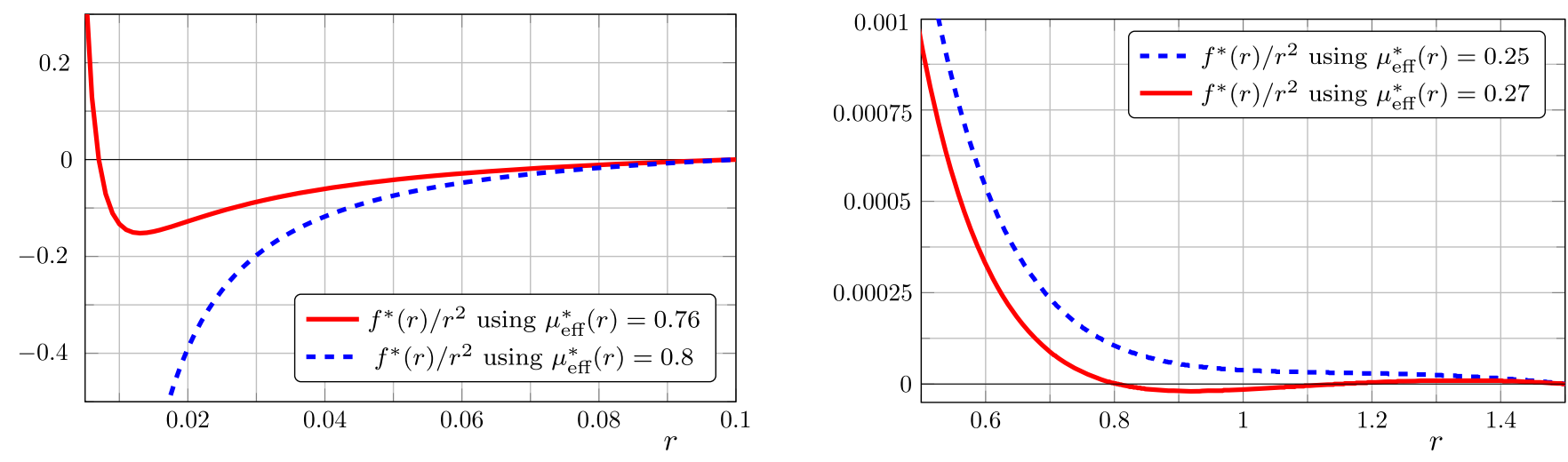

FIG. 4. Fixed point background potential for different constant input values of $\mu_{\mathrm{eff}}^{*}(r)$. The minimum that corresponds to the solution of the background equation of motion is at $r>0$ for $\mu_{\mathrm{eff}}^{*}(r) \lesssim 0.77$, while for $\mu_{\mathrm{eff}}^{*}(r) \gtrsim 0.77$ it is at $r<0$ (left panel). For $\mu_{\mathrm{eff}}^{*}(r) \lesssim 0.25$ the minimum vanishes completely, while for $\mu_{\mathrm{eff}}^{*}(r)=0.26$ the minimum is located at $r_{0}=1.1$ (right panel).

that solutions exist for positive curvature when $0.255 \lesssim$ $\mu_{\mathrm{eff}}^{*} \lesssim 0.77$ and for negative curvature for $\mu_{\mathrm{eff}}^{*} \gtrsim 0.77$. For $\mu_{\mathrm{eff}}^{*} \lesssim 0.255$ there are no solutions. The transition of the minimum from positive to negative curvature is depicted in the left panel of Fig. 4 while the full disappearance of the minimum is depicted in the right panel. The computed value of $\mu_{0}^{*}=-0.38$, see (35), is far away from the value where the solution appears. Thus we conclude that the absence of a minimum in the background potential in our full pure gravity computation is rather stable with respect to changes in the truncation.

\section{Dependence on matter}

Matter can potentially have a significant influence on the properties of the UV fixed point, see e.g. [7,13,59,63-65]. In the present work matter influences the existence of a minimum in the background potential in two ways: On the one hand it has an influence on the fixed point values of the fluctuation couplings, where in particular the influence on $\mu_{\mathrm{eff}}^{*}(r)$ is important. On the other hand it has a direct influence on the background potential via the background matter loops. Both these effects have been studied in a fluctuation computation on a flat background, see [7] for scalars and fermions and [13] for gauge bosons. Consequently we adapt the analysis to curved backgrounds under the assumption that the effective graviton mass parameter $\mu_{\text {eff }}(r)$ remains a almost curvature independent in these extended systems, similar to the results displayed in Sec. VA.

Combining the results of $[7,13]$ for standard model matter content $\left(N_{s}=4, N_{f}=22.5\right.$, and $\left.N_{v}=12\right)$ gives a UV fixed point at

$$
\left(g_{0}^{*}, \lambda_{3,0}^{*}, \mu_{0}^{*}\right)_{\mathrm{SM}}=(0.17,0.15,-0.71),
$$

and will be reported in [124]. For the present analysis only the value $\mu_{0, \mathrm{SM}}^{*}$ is important since we now use $\mu_{\mathrm{eff}}^{*}(r)=$ $\mu_{0, \mathrm{SM}}^{*}$ as an input for the background potential. The matter content seemingly pushes $\mu_{\mathrm{eff}}^{*}$ in the wrong direction, cf. Fig. 4. However, the matter content has also a huge influence on the background equations. The combined result is displayed in Fig. 5. Indeed we find a minimum in the background potential at small curvature, $r_{0}=0.11$. Also in the Einstein-Hilbert approximation, i.e. $\mu^{*}(r)=\mu_{0, \mathrm{SM}}^{*}$, we find a minimum at $r_{0, \mathrm{EH}}=0.05$. With standard model matter content the full solution and the Einstein-Hilbert approximation are very similar. This comes as a surprise as the difference was rather significant without matter content, cf. Fig. 3.

\section{Quantum equation of motion}

In this section we evaluate the graviton one-point function and thus look for solutions to the quantum EoM (7). As discussed in Sec. III A the solution to this equation leads to self-consistent backgrounds that improve the convergence of the Taylor series. Moreover, it has been

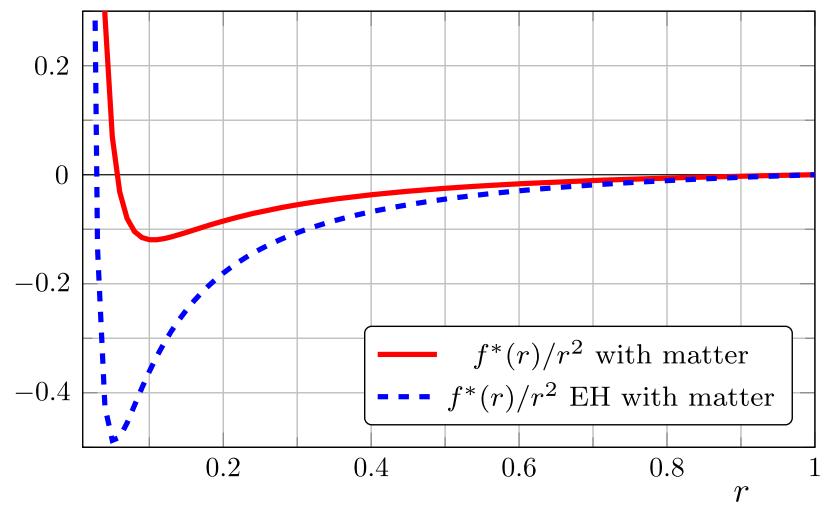

FIG. 5. Depicted is the fixed point background potential if standard model matter content is included. In the full solution as well as in the Einstein-Hilbert solution we find a minimum at small background curvature, $r_{0}=0.11$ and $r_{0, \mathrm{EH}}=0.05$, respectively, which corresponds to the solution of the background equation of motion. 


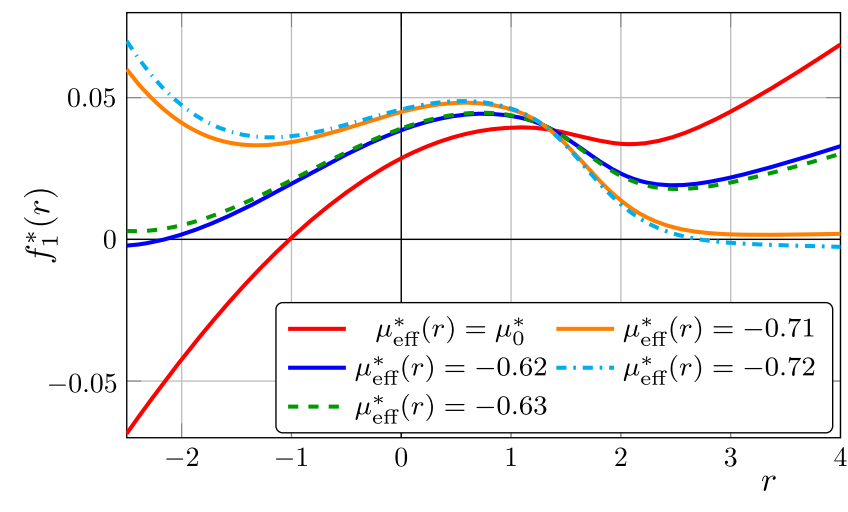

FIG. 6. Shown is the fixed point function $f_{1}^{*}(r)$ for different constant input values of $\mu_{\mathrm{eff}}^{*}(r)$. The zeros in these functions correspond to solutions to the quantum equations of motion (7). Our best result $\mu_{\mathrm{eff}}^{*}(r)=\mu_{0}^{*}=-0.38$ has a solution at negative curvature, $r_{0}=-1.0$.

also argued there that the quantum EoM in the limit $k \rightarrow \infty$ should be seen as an estimate for the solution of the UV EoM in the physical limit $k \rightarrow 0$ where background and quantum EoM agree due to background independence.

Within the present setup the only invariant linear in the fluctuation field is given by $f_{1}(r) h^{\mathrm{tr}}$ with some function $f_{1}$ that is determined by the fluctuation couplings. An invariant linear in the transverse traceless mode does not exist due to our restriction to a spherical background and thus the absence of terms like $r^{\mu \nu} h_{\mu \nu}^{\mathrm{tt}}$. Consequently we evaluate (7) with a projection on the trace mode of the graviton.

In straight analogy to the background EoM (42) we parametrize the one-point function by

$\Gamma^{\left(h_{\mathrm{tr}}\right)}[\bar{g}, 0]=\int \mathrm{d}^{4} x \sqrt{\bar{g}} k^{3} f_{1}\left(\bar{R} / k^{2}\right)=\frac{384 \pi^{2}}{k r^{2}} f_{1}(r)$.

We denote again the right-hand side by $\mathcal{F}_{1}(r, \mu(r))$. This time, however, we obtain at a different differential equation for $f_{1}$ due to the different mass-dimensions of $\bar{g}$ and $h$. Thus $f_{1}$ obeys the fixed point equation

$$
\frac{384 \pi^{2}}{r^{2}}\left(3 f_{1}^{*}(r)-2 r f_{1}^{* \prime}(r)\right)=\mathcal{F}_{1}\left(r, \mu^{*}(r)\right) .
$$

We solve this equation with the initial condition that $f_{1}^{*}(r)$ is finite at $r=0$. Consequently we combine a heat kernel expansion around $r=0$ up to the order $r^{3}$ with a spectral sum evaluation for large, positive curvature. For results at negative curvature we rely on the heat kernel expansion, but from a comparison of the heat kernel results with the spectral sum at positive curvature we can estimate the radius of convergence of the heat kernel. We estimate the latter by the range where the relative change is in the sub percent regime. We find that the radius of convergence is approximately given by $r_{\text {conv }} \approx 1$. The radius of convergence increases for larger $\mu_{\mathrm{eff}}^{*}(r)$.

The resulting fixed point functions $f_{1}^{*}(r)$ are shown in Fig. 6. For our best result $\mu_{\mathrm{eff}}^{*}(r)=\mu_{0}^{*}=-0.38, f_{1}^{*}(r)$ has a root at negative curvature, $r_{0}=-1.0$, which corresponds to a solution to the quantum EoM. The result lies within the radius of convergence of the heat-kernel expansion and thus we consider it trustworthy.

We again check the stability of the solution by treating $\mu_{\mathrm{eff}}^{*}(r)$ as a constant free input parameter. For more positive values, $\mu_{\mathrm{eff}}^{*}>\mu_{0}^{*}$, the root of $f_{1}^{*}(r)$ moves toward larger curvature, but always remains negative. In the limit $\mu_{\mathrm{eff}}^{*} \rightarrow \infty$ the root is located at $r_{0}=-0.42$. For more negative values, $\mu_{\mathrm{eff}}^{*}<\mu_{0}^{*}$ the root of $f_{1}^{*}(r)$ moves toward smaller curvature and eventually the root disappears at $\mu_{\text {eff }}^{*}=-0.62$, cf. Fig. 6 . This result has to be taken very careful since at $\mu_{\mathrm{eff}}^{*}=-0.62$ the root is located at $r_{0}=$ -2.2 and thus lies outside of the radius of convergence of heat kernel. At $\mu_{\text {eff }}^{*}=-0.71$ a new solution appears at positive curvature, $r_{0}=2.7$. This root remains also for more negative values of $\mu_{\mathrm{eff}}^{*}$ until the pole at $\mu_{\mathrm{eff}}^{*}=-1$. The roots at positive curvature are obtained with the spectral sum and thus do not rely on the radius of convergence of the heat kernel.

We have visualized the existence of a solution to the background and quantum EoM in Fig. 7. The quantum EoM has almost always a solution, only in the range $-0.71<\mu_{\mathrm{eff}}^{*}<-0.62$ no solution exists. This range may even disappear with better truncations or an improved computation at large negative curvature. The background EoM on the other hand only allows for a solution for $\mu_{\mathrm{eff}}^{*}>0.26$, and thus in a region that is very unusual for pure gravity computations.

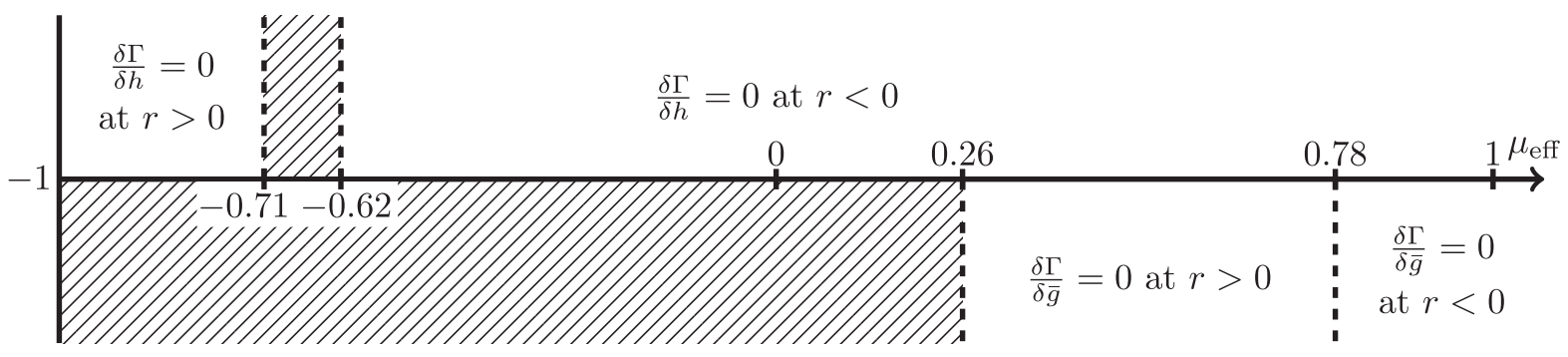

FIG. 7. Visualization of the existence of a solution to the background and quantum equation of motion in dependence on the parameter $\mu_{\mathrm{eff}}$. Solutions at positive curvature $(r>0)$ and negative curvature $(r<0)$ are distinguished. 


\section{SUMMARY AND OUTLOOK}

In this work we have developed an approach to asymptotically safe gravity with nontrivial backgrounds. As a first application of the novel approach we computed the $f(R)$-potential and discussed solutions of the equations of motion.

We have also given a discussion of functional approaches to quantum gravity that take into account the necessary background independence of the theory. We have discussed, for the first time in quantum gravity, that background independence and diffeomorphism invariance can be achieved iteratively in any approximation scheme, based on a similar argument in non-Abelian gauge theories, see Sec. II. We have also emphasized the relevance of aiming for solutions that satisfy all functional relations. We have argued that this is tightly bound to the question of unitarity.

The approach is based on a vertex expansion of the effective action about nontrivial backgrounds, which at present are restricted to constantly curved backgrounds. Our explicit results are based on a truncation that includes the flow of the graviton two- and three-point function and thus the couplings $g, \lambda_{3}$, and $\mu$. The construction of an approximate momentum space, cf. (18), allowed us to evaluate these couplings without a derivative expansion in momentum $p$ or curvature $r$. In this work we focused on the curvature dependence and thus all couplings are functions of the curvature, $g(r), \lambda_{3}(r)$, and $\mu(r)$. The flow equations for these coupling functions were obtained with spectral sums on a sphere. The results are smoothly connected to known results at vanishing background curvature with heatkernel methods.

As one main result we found UV fixed point functions that confirm the asymptotic safety of the present system. Interestingly, the effective fixed point couplings, $\lambda_{3, \text { eff }}^{*}(r)$ and $\mu_{\mathrm{eff}}^{*}(r)$, cf. (37), turned out to be almost curvature independent over the investigated range: the couplings counterbalance the explicit curvature dependence of the $n$-point functions.

We have also discussed the background and the quantum equation of motion, (6) and (7), in Sec. III. At $k=0$, their solutions agree due to background independence. In turn, at finite $k$ the solutions to background and quantum equations of motion differ due to a regulator contribution to the modified Nielsen identity. This signals the breaking of background independence in the presence of the cutoff. We have argued in the present work that at finite cutoff it is the solution of the quantum equation of motion that relates directly to the physical solution of the equation of motion at vanishing cutoff.

We explicitly evaluated both equations of motion with the UV fixed point functions and indeed found different solutions: The background equation of motion does not feature a solution. Only with standard model matter content a solution at small curvature is present. The quantum equation of motion exhibits already a solution at negative curvature without any matter content. We have checked the stability of these statements by scanning for solutions in the parameter $\mu_{\mathrm{eff}}^{*}$. The background equation of motion without matter features a solution only for very large values of $\mu_{\mathrm{eff}}^{*}$, far away from most values observed in pure quantum gravity truncations. On the other hand the quantum equation of motion has a solution for almost all $\mu_{\text {eff }}^{*}$. This indicates that the existence of a solution seems to be robust with respect to changes in the truncation. We have visualized this behavior in Fig. 7.

The discussion of the equation of motion leads us directly to a specific observable: the effective action, evaluated on the equation of motion. In standard quantum field theories this is the free energy, and it is gauge and parametrization independent. For the present approach this is discussed in Sec. III A. We therefore expect only a mild dependence on these choices within sensible approximations to the full effective action. Indeed, this has been observed in the background field approximation $[125,126]$. It would be interesting to see whether this property is also holds in the present approach that goes beyond the background field approximation. At finite cutoff this investigation can be done by studying the gauge and parametrization independence of the effective action evaluated on the quantum equation of motion. This will be discussed elsewhere.

Possible improvements of the present work involve the inclusion of momentum- and curvature-dependent anomalous dimensions as well as the inclusion of further $R^{2}$ - and $R_{\mu \nu}^{2}$-tensor structures in the generating vertices. It would be very interesting to extend the present work to more general backgrounds. Moreover, the present approach also allows us to take the limit $k \rightarrow 0$. This allows us, for the first time, to directly discuss asymptotically safe physics directly for the physically relevant cutoff scale $k=0$. Applications range from asymptotically safe cosmology with the quantum $f(R)$ potential as well as the UV-behavior and phenomenology of the asymptotically safe (extensions of the) standard model. We hope to report on these applications in the near future.

\section{ACKNOWLEDGMENTS}

We thank B. Knorr, S. Lippoldt, T. Morris and C. Wetterich for discussions. NC acknowledges funding from the DFG under the Emmy Noether program, Grant No. Ei1037-1, and MR from IMPRS-PTFS. This work is supported by the Helmholtz Alliance HA216/EMMI and by ERC-AdG-290623. It is part of and supported by the DFG Collaborative Research Centre SFB 1225 (ISOQUANT).

\section{APPENDIX A: PROPAGATOR}

We use the standard York decomposition to invert the two-point functions. The York-decomposition for the graviton is given by 


$$
h_{\mu \nu}=h_{\mu \nu}^{\mathrm{tt}}+\frac{1}{d} \bar{g}_{\mu \nu} h^{\mathrm{tr}}+2 \bar{\nabla}_{(\mu} \xi_{\nu)}+\left(\bar{\nabla}_{\mu} \bar{\nabla}_{\nu}-\frac{\bar{g}_{\mu \nu}}{d} \bar{\nabla}^{2}\right) \sigma .
$$

and for the ghost by

$$
c_{\mu}=c_{\mu}^{T}+\bar{\nabla}_{\mu} \eta
$$

and analogously for the antighost. With the field redefinitions according to $[24,44,51]$

$$
\begin{aligned}
\xi^{\mu} & \rightarrow \frac{1}{\sqrt{\bar{\Delta}-\frac{\bar{R}}{4}}} \xi^{\mu}, \\
\sigma & \rightarrow \frac{1}{\sqrt{\bar{\Delta}^{2}-\bar{\Delta} \frac{\bar{R}}{3}}} \sigma, \\
\eta & \rightarrow \frac{1}{\bar{\Delta}} \eta,
\end{aligned}
$$

we cancel the nontrivial Jacobians and achieve that all field modes have the same mass dimension. We choose the gauge $\alpha=\beta=0$ and choose the regulator proportional to the two-point function

$$
R_{k}=\left.\Gamma^{(2)}\right|_{\Lambda \rightarrow 0, R \rightarrow 0} \cdot r_{k}\left(p^{2}\right)
$$

Here and in the following in this Appendix, $p^{2}$ always refers to the dimensionless spectral values of the scalar Laplacian. For the regulator shape function $r_{k}$, we choose an exponential regulator

$$
r_{k}(x)=\frac{\mathrm{e}^{-x^{2}}}{x}
$$

The propagator has the form

$$
G=\frac{32 \pi}{Z_{h}}\left(\begin{array}{cccc}
\frac{1}{p^{2}\left(1+r_{k}\left(p^{2}\right)\right)+\mu+\frac{2}{3} r} & 0 & 0 & 0 \\
0 & 0 & 0 & 0 \\
0 & 0 & \frac{-\frac{8}{3}}{p^{2}\left(1+r_{k}\left(p^{2}\right)\right)+\frac{2}{3} \mu} & 0 \\
0 & 0 & 0 & 0
\end{array}\right),
$$

where the first entry is the transverse traceless mode and the third entry is the trace mode. All other modes vanish due to Landau gauge, $\alpha=0$. Furthermore, we get the following expressions for the background flow of the different graviton modes, where still the spectral sum/integral or heat-kernel expansion has to be performed, $\frac{1}{2} \operatorname{Tr}\left[G \partial_{t} R\right]_{h_{\mathrm{tt}}}=\frac{r^{2}}{768 \pi^{2}} \frac{p^{2}\left(\partial_{t} r_{k}\left(p^{2}\right)-\eta_{h} r_{k}\left(p^{2}\right)\right)}{p^{2}\left(1+r_{k}\left(p^{2}\right)\right)+\mu+\frac{2}{3} r}$,

$$
\frac{1}{2} \operatorname{Tr}\left[G \partial_{t} R\right]_{\xi}=\frac{r^{2}}{768 \pi^{2}} \frac{p^{2}\left(\partial_{t} r_{k}\left(p^{2}\right)-\eta_{h} r_{k}\left(p^{2}\right)\right)}{p^{2}\left(1+r_{k}\left(p^{2}\right)\right)-\frac{1}{4} r},
$$

$\frac{1}{2} \operatorname{Tr}\left[G \partial_{t} R\right]_{h_{\mathrm{tr}}}=\frac{r^{2}}{768 \pi^{2}} \frac{p^{2}\left(\partial_{t} r_{k}\left(p^{2}\right)-\eta_{h} r_{k}\left(p^{2}\right)\right)}{p^{2}\left(1+r_{k}\left(p^{2}\right)\right)+\frac{2}{3} \mu}$,

$\frac{1}{2} \operatorname{Tr}\left[G \partial_{t} R\right]_{\sigma}=\frac{r^{2}}{768 \pi^{2}} \frac{p^{2}\left(\partial_{t} r_{k}\left(p^{2}\right)-\eta_{h} r_{k}\left(p^{2}\right)\right)}{p^{2}\left(1+r_{k}\left(p^{2}\right)\right)-\frac{1}{3} r}$.

And for the ghosts

$$
\begin{aligned}
-\operatorname{Tr}\left[G \partial_{t} R\right]_{c} & =-\frac{r^{2}}{384 \pi^{2}} \frac{p^{2}\left(\partial_{t} r_{k}\left(p^{2}\right)-\eta_{c} r_{k}\left(p^{2}\right)\right)}{p^{2}\left(r_{k}\left(p^{2}\right)+1\right)-\frac{r}{4}}, \\
-\operatorname{Tr}\left[G \partial_{t} R\right]_{\eta} & =-\frac{r^{2}}{384 \pi^{2}} \frac{p^{2}\left(\partial_{t} r_{k}\left(p^{2}\right)-\eta_{c} r_{k}\left(p^{2}\right)\right)}{p^{2}\left(r_{k}\left(p^{2}\right)+1\right)-\frac{1}{3} r} .
\end{aligned}
$$

\section{APPENDIX B: FLOW EQUATIONS}

The flow equation for the transverse traceless part of the graviton two-point function is given by

$$
\frac{1}{32 \pi} \partial_{t}\left(Z_{h} k^{2}\left(\mu+p^{2}+\frac{2}{3} r\right)\right)=k^{2} Z_{h} \text { Flow }_{\mathrm{tt}}^{(2 h)}\left(p^{2}\right) .
$$

Here we suppressed the dependences of the couplings on e.g. background curvature $r$ or spectral values $p^{2}$ to improve readability. All dependences are as in Sec. IV B. The expression Flow is used as in [11] and stands here and in the following for the dimensionless right-hand side of the flow equation divided by appropriate powers of the wave function renormalizations. The superscript specifies the $n$-point function, while the subscript refers to the tensor projection.

From (B1) we obtain the flow equation for the transverse traceless graviton mass parameter

$$
\partial_{t} \mu=\left(\eta_{h}-2\right) \mu+\frac{2}{3} \eta_{h} r+2 r \mu^{\prime}+32 \pi \operatorname{Flow}_{\mathrm{tt}}^{(2 h)}\left(p^{2}=0\right),
$$

where the $/$ refers to a derivative with respect to $r$. The graviton three-point function is projected in straight analogy to the flat computation [6]. We focus on the transverse traceless part and define the two projection operators $\Pi_{\Lambda}$ and $\Pi_{G}$ as

$$
\begin{aligned}
& \Pi_{\Lambda}=\Pi_{\mathrm{tt}}^{3} S_{\mathrm{EH}}^{(3 h)}\left(p^{2}=0, r=0\right), \\
& \Pi_{G}=\Pi_{\mathrm{tt}}^{3} S_{\mathrm{EH}}^{(3 h)}(\Lambda=0, r=0),
\end{aligned}
$$


which we use for the projection on $\lambda_{3}$ and $g$, respectively. The resulting flow equations are

$$
\begin{aligned}
& \partial_{t}\left(Z_{h}^{3 / 2} k^{2} \sqrt{g}\left(\frac{5}{2304} r+\frac{5}{192} \lambda_{3}-\frac{9}{4096} p^{2}\right)\right) \\
& \quad=k^{2} Z_{h}^{3 / 2} \operatorname{Flow}_{\Lambda}^{(3 h)}\left(p^{2}\right), \\
& \partial_{t}\left(Z_{h}^{3 / 2} k^{2} \sqrt{g}\left(-\frac{3}{8192} r-\frac{9}{4096} \lambda_{3}+\frac{171}{32768} p^{2}\right)\right) \\
& \quad=k^{2} Z_{h}^{3 / 2} \operatorname{Flow}_{G}^{(3 h)}\left(p^{2}\right) .
\end{aligned}
$$

The flow of $\lambda_{3}$ is extracted at vanishing spectral value $p^{2}=0$, while the flow of $g$ is extracted with a derivative with respect to the dimensionless spectral value $p^{2}$ at $p=0$. The result is

$$
\begin{aligned}
\partial_{t} \lambda_{3}= & -2 \lambda_{3}+2 r \lambda_{3}^{\prime}+\left(\frac{3}{2} \eta_{h}+\frac{12 g-\partial_{t} g+2 r g^{\prime}}{g}\right)\left(\lambda_{3}+\frac{1}{12} r\right) \\
& +\frac{3}{80} \frac{(32 \pi)^{2}}{\sqrt{g} k} \operatorname{Flow}_{\Lambda}^{(3 h)}\left(p^{2}=0\right), \\
\partial_{t} g= & 2 g+2 r g^{\prime}+3 \eta_{h} g-\frac{24}{19}\left(\left.\partial_{p^{2}} \eta_{h}\right|_{p^{2}=0}\right)\left(\lambda_{3}+\frac{1}{6} r\right) g \\
& +\frac{64}{171}(32 \pi)^{2} \sqrt{g} k \partial_{p^{2}} \text { Flow }\left._{G}^{(3 h)}\right|_{p^{2}=0} .
\end{aligned}
$$

The derivation of the flow equations in this section required contractions of very large tensor structures. These contractions were computed with the help of the symbolic manipulation system FORM $[127,128]$. We furthermore used the Mathematica packages XPERT [129] for the generation of vertex functions, and the FORMTRACER [130] to trace diagrams.

\section{APPENDIX C: CHECK OF APPROXIMATIONS}

In Sec. IVA we have explained that all vertices in a curved background contain uncontracted covariant derivatives. We have circumvented this issue by using the approximation displayed in (18). This problem reoccurs during the contraction of the diagrams, since the usual York-decomposition projection operators $\Pi_{i}$ are needed, with $i \in\{\mathrm{tt}, \mathrm{tr}, \ldots\}$. The projection operators are functions of the background Laplacian and the background covariant derivative $\Pi_{i}(\bar{\Delta}, \bar{\nabla})$, where the latter covariant derivatives are again approximated by (17) and (18). This however causes us to mix up the different spin Laplacians, spin-two $\Delta_{2}$ and spin-zero $\Delta_{0}$. Other Laplacians do not occur since the graviton propagator only has a nonvanishing transverse traceless and trace mode. In the this work we choose to use the spin-zero Laplacian.

For the background flow this mixing of Laplacians does not occur since the propagator is not a function of the covariant derivative. Hence we use the background flow to estimate the error of our approximation. Here we focus on the transverse traceless and the trace part since these are the relevant modes in the fluctuation computation. The exact result with our regulator is given by

$$
\begin{aligned}
\operatorname{Tr}\left[G \partial_{t} R\right]_{\mathrm{tt}, \mathrm{tr}}= & \sum_{\ell=2}^{\ell_{\max }} m_{2}(\ell)\left(G \partial_{t} R\right)_{\mathrm{tt}}\left(\Delta_{2}(l)\right) \\
& +\sum_{\ell=0}^{\ell_{\max }} m_{0}(\ell)\left(G \partial_{t} R\right)_{\mathrm{tr}}\left(\Delta_{0}(l)\right)
\end{aligned}
$$

while we compare it to the approximations

$$
\begin{aligned}
(\mathrm{C} 1) & \approx \sum_{\ell=0}^{\ell_{\max }} m_{0}(\ell)\left(5\left(G \partial_{t} R\right)_{\mathrm{tt}}+\left(G \partial_{t} R\right)_{\mathrm{tr}}\right)\left(\Delta_{0}(l)\right) \\
& \approx \sum_{\ell=2}^{\ell_{\max }} m_{0}(\ell)\left(5\left(G \partial_{t} R\right)_{\mathrm{tt}}+\left(G \partial_{t} R\right)_{\mathrm{tr}}\right)\left(\Delta_{0}(l)\right) \\
& \approx \sum_{\ell=2}^{\ell_{\max }} m_{2}(\ell)\left(\left(G \partial_{t} R\right)_{\mathrm{tt}}+\frac{1}{5}\left(G \partial_{t} R\right)_{\mathrm{tr}}\right)\left(\Delta_{2}(l)\right) .
\end{aligned}
$$

Here $\ell_{\max }$ is chosen such that the trace is fully converged in the investigated curvature range and the factors 5 and $\frac{1}{5}$ appear due to the five transverse traceless modes compared to the one trace mode.

The results are shown in Fig. 8 in the left panel. For small background curvature $r$ all results agree qualitatively well. For large background curvature the difference is becoming more significant. This can be easily understood: in the exact result $(\mathrm{C} 1)$ only the trace mode is equipped with a zero mode, while in the first approximation (C2) all modes are equipped with a zero mode. In contrast in the second and third approximation, (C3) and (C4), no mode is equipped with a zero mode. The zero modes dominate for large curvature and thus it is clear that the approximation fails in this regime.

In other words, the symmetrized products of covariant derivatives in the projectors are effectively commuting in our approximation. The transverse traceless projection basically traces out the degrees of freedom of the transverse traceless mode and leaves us with a scalar quantity. With this approximation, there is an ambiguity related to the Laplace operator, which can be chosen as the spin-zero or spin-two Laplacian. As already mentioned we choose to use the spin-zero Laplacian without zero modes, i.e. approximation $(\mathrm{C} 3)$.

In the right panel of Fig. 8 we compare these different choices for one particular diagram of the graviton two-point function, where the exact result is not available within our truncation. We observe that the results are almost identical for small curvature, i.e. $r<2$. For $r>2$ the results differ qualitatively due to the different treatment of the zero 

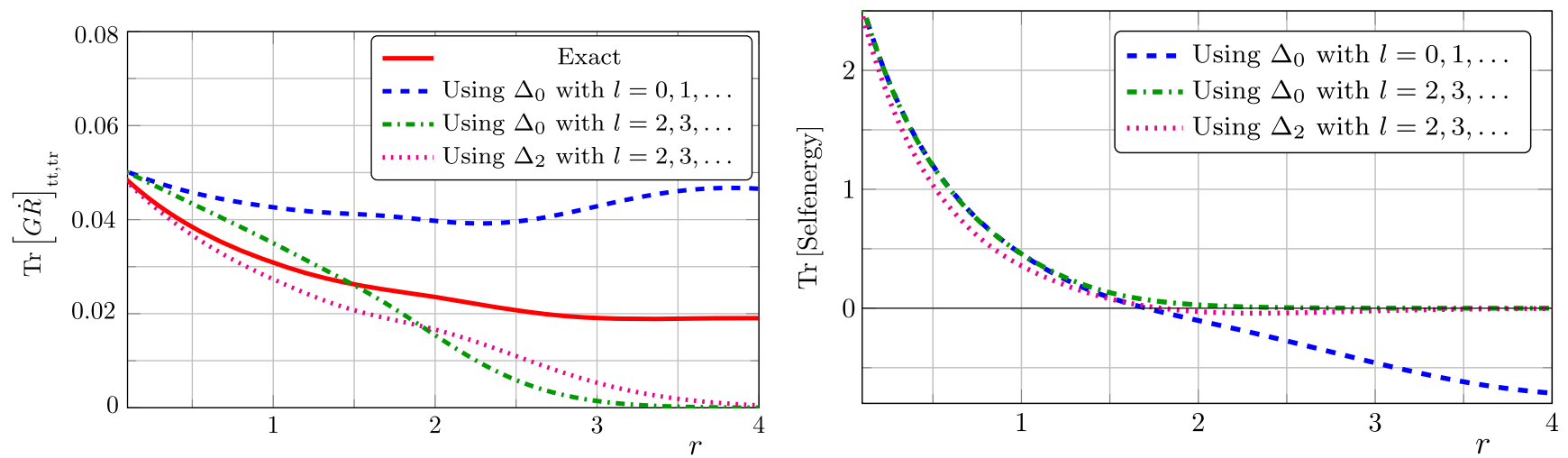

FIG. 8. Comparison of the trace evaluation using different Laplacians and starting with different eigenvalues. In particular we compare the spin-two Laplacian $\Delta_{2}$ and the spin-zero Laplacian $\Delta_{0}$ and further we start once from the zero mode and once start from the $l=2$ mode. In the left panel we display the background flow $\operatorname{Tr}\left[G \partial_{t} R\right]$ of the combined transverse traceless and trace mode, where also the exact solution is computed. In the right panel we display the self-energy diagram of the two-point function, which is the second diagram in Fig. 1. From these results we infer that this particular approximation is qualitatively reliable in the range $r<2$.

modes. We conclude that the validity of our approximation is bound by $r<2$.

\section{APPENDIX D: INSENSITIVITY ON INITIAL CONDITIONS}

As explained in Sec. IVC we have to give initial conditions to the beta function since they are the firstorder linear differential equations. In principle the initial condition has to be given at vanishing curvature $r=0$ since there are the divergences of the differential equations. However the spectral sum converges only point wise and the number of modes that have to be included grows exponentially towards $r \rightarrow 0$. Consequently we give the initial conditions at some finite $r_{\text {start }}$ that should be close to $r=0$. The value there is obtained by expanding the heat kernel expansion (32). One can then check that the spectral sum and heat kernel agree in the small background curvature regime where both methods converge $[122,123]$. In this Appendix we discus the sensitivity of the fixed point functions to the choice of $r_{\text {start }}$.

The initial condition for some coupling $g_{i}$ is determined from the zero and first order of the heat-kernel expansion around $r=0$, i.e. by $g_{i}^{*}\left(r_{\text {start }}\right)=g_{i, 0}^{*}+r_{\text {start }} g_{i, 1}^{*}$ where $g_{i, 0}^{*}$ and $g_{i, 1}^{*}$ are determined by the heat-kernel computation and the solutions are displayed in (35) and (39). On the one hand the quality of this initial condition gets worse for large $r_{\text {start }}$ since this is a linear approximation of the curvature dependence of the couplings. On the other hand the quality also gets worse for too small $r_{\text {start }}$ since we are too close to the singularity at $r=0$. Consequently we have to find a region in between where the fixed point functions for the couplings are stable against small variations of $r_{\text {start }}$.

From the chosen $r_{\text {start }}$ we integrate the differential equations upwards to large $r$. Integrating down would quickly run into the singularity at $r=0$. In Fig. 9 we display the resulting
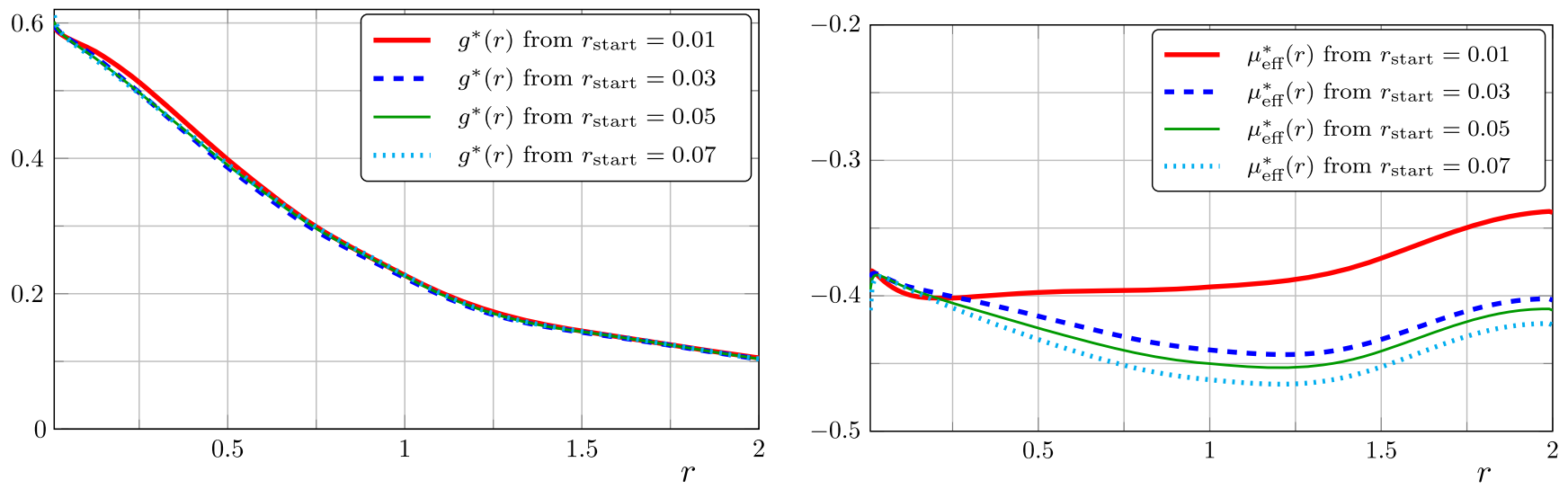

FIG. 9. Comparison of fixed point functions with initial condition at different curvature values $r_{\text {start }} \in\{0.01,0.03,0.05,0.07\}$. In the left panel we compare the fixed point functions of the Newton's coupling $g(r)$ and in the right panel the effective graviton mass parameter $\mu_{\text {eff }}(r)=\mu(r)+\frac{2}{3} r$. Both fixed point functions show only a small dependence on the initial condition. All initial conditions are determined by $g_{i}^{*}\left(r_{\text {start }}\right)=g_{i, 0}^{*}+r_{\text {start }} g_{i, 1}^{*}$, where the zeroth and linear order in $r$ of the couplings are given by (35) and (39). 
fixed point functions for $g^{*}(r)$ and $\mu_{\mathrm{eff}}^{*}(r)$ for different choices of $r_{\text {start }} \in\{0.01,0.03,0.05,0.07\}$. We observe that the fixed point functions for $g^{*}(r)$ (left panel of Fig. 9) agree almost perfectly for all chosen start values. Only for $r_{\text {start }}=0.01 \mathrm{we}$ observe a tiny deviation. For the fixed point functions of $\mu_{\text {eff }}^{*}(r)$ (right panel of Fig. 9) we observe larger, but still small deviations. Again for $r_{\text {start }}=0.01$ the deviations are the largest. We conclude that the this start value is too close to the singularity at $r=0$. The results in this work were computed with $r_{\text {start }}=0.03$.
[1] S. Weinberg, General Relativity: An Einstein Centenary Survey, edited by S. W. Hawking and W. Israel (Cambridge University Press, Cambridge, England, 1979), p. 790.

[2] M. Reuter, Phys. Rev. D 57, 971 (1998).

[3] N. Christiansen, D. F. Litim, J. M. Pawlowski, and A. Rodigast, Phys. Lett. B 728, 114 (2014).

[4] A. Codello, G. D’Odorico, and C. Pagani, Phys. Rev. D 89, 081701 (2014).

[5] N. Christiansen, B. Knorr, J. M. Pawlowski, and A. Rodigast, Phys. Rev. D 93, 044036 (2016).

[6] N. Christiansen, B. Knorr, J. Meibohm, J. M. Pawlowski, and M. Reichert, Phys. Rev. D 92, 121501 (2015).

[7] J. Meibohm, J. M. Pawlowski, and M. Reichert, Phys. Rev. D 93, 084035 (2016).

[8] J. Meibohm and J. M. Pawlowski, Eur. Phys. J. C 76, 285 (2016).

[9] T. Henz, J. M. Pawlowski, and C. Wetterich, Phys. Lett. B 769, 105 (2017).

[10] N. Christiansen, arXiv:1612.06223.

[11] T. Denz, J. M. Pawlowski, and M. Reichert, arXiv:1612 .07315 .

[12] B. Knorr and S. Lippoldt, Phys. Rev. D 96, 065020 (2017).

[13] N. Christiansen, D. F. Litim, J. M. Pawlowski, and M. Reichert, arXiv:1710.04669.

[14] B. Knorr, arXiv:1710.07055.

[15] I. Donkin and J. M. Pawlowski, arXiv:1203.4207.

[16] T. R. Morris, J. High Energy Phys. 11 (2016) 160.

[17] R. Percacci and G. P. Vacca, Eur. Phys. J. C 77, 52 (2017).

[18] E. Manrique and M. Reuter, Ann. Phys. (Amsterdam) 325, 785 (2010).

[19] E. Manrique, M. Reuter, and F. Saueressig, Ann. Phys. (Amsterdam) 326, 440 (2011).

[20] E. Manrique, M. Reuter, and F. Saueressig, Ann. Phys. (Amsterdam) 326, 463 (2011).

[21] D. Becker and M. Reuter, Ann. Phys. (Amsterdam) 350, 225 (2014).

[22] S. Falkenberg and S. D. Odintsov, Int. J. Mod. Phys. A 13, 607 (1998).

[23] M. Reuter and F. Saueressig, Phys. Rev. D 65, 065016 (2002).

[24] O. Lauscher and M. Reuter, Phys. Rev. D 65, 025013 (2001).

[25] O. Lauscher and M. Reuter, Phys. Rev. D 66, 025026 (2002).

[26] D. F. Litim, Phys. Rev. Lett. 92, 201301 (2004).

[27] P. Fischer and D. F. Litim, Phys. Lett. B 638, 497 (2006).

[28] A. Codello and R. Percacci, Phys. Rev. Lett. 97, 221301 (2006).
[29] P. F. Machado and F. Saueressig, Phys. Rev. D 77, 124045 (2008).

[30] A. Codello, R. Percacci, and C. Rahmede, Ann. Phys. (Amsterdam) 324, 414 (2009).

[31] A. Eichhorn, H. Gies, and M. M. Scherer, Phys. Rev. D 80, 104003 (2009).

[32] D. Benedetti, P. F. Machado, and F. Saueressig, Mod. Phys. Lett. A 24, 2233 (2009).

[33] A. Eichhorn and H. Gies, Phys. Rev. D 81, 104010 (2010).

[34] K. Groh and F. Saueressig, J. Phys. A 43, 365403 (2010).

[35] E. Manrique, S. Rechenberger, and F. Saueressig, Phys. Rev. Lett. 106, 251302 (2011).

[36] D. Benedetti and F. Caravelli, J. High Energy Phys. 06 (2012) 017; 10 (2012) 157(E).

[37] J. A. Dietz and T. R. Morris, J. High Energy Phys. 01 (2013) 108.

[38] K. Falls, D. Litim, K. Nikolakopoulos, and C. Rahmede, arXiv:1301.4191.

[39] K. Falls, D. F. Litim, K. Nikolakopoulos, and C. Rahmede, Phys. Rev. D 93, 104022 (2016).

[40] K. Falls, Phys. Rev. D 92, 124057 (2015).

[41] A. Eichhorn, J. High Energy Phys. 04 (2015) 096.

[42] K. Falls, arXiv:1503.06233.

[43] M. Demmel, F. Saueressig, and O. Zanusso, J. High Energy Phys. 08 (2015) 113.

[44] H. Gies, B. Knorr, and S. Lippoldt, Phys. Rev. D 92 , 084020 (2015).

[45] H. Gies, B. Knorr, S. Lippoldt, and F. Saueressig, Phys. Rev. Lett. 116, 211302 (2016).

[46] J. Biemans, A. Platania, and F. Saueressig, Phys. Rev. D 95, 086013 (2017).

[47] K. Falls, Phys. Rev. D 96, 126016 (2017).

[48] Y. Hamada and M. Yamada, J. High Energy Phys. 08 (2017) 070.

[49] S. Gonzalez-Martin, T. R. Morris, and Z. H. Slade, Phys. Rev. D 95, 106010 (2017).

[50] D. Becker, C. Ripken, and F. Saueressig, J. High Energy Phys. 12 (2017) 121.

[51] D. Dou and R. Percacci, Classical Quantum Gravity 15, 3449 (1998).

[52] R. Percacci and D. Perini, Phys. Rev. D 67, 081503 (2003).

[53] S. Folkerts, D. F. Litim, and J. M. Pawlowski, Phys. Lett. B 709, 234 (2012).

[54] U. Harst and M. Reuter, J. High Energy Phys. 05 (2011) 119.

[55] A. Eichhorn and H. Gies, New J. Phys. 13, 125012 (2011).

[56] A. Eichhorn, Phys. Rev. D 86, 105021 (2012).

[57] P. Donà and R. Percacci, Phys. Rev. D 87, 045002 (2013). 
[58] T. Henz, J. M. Pawlowski, A. Rodigast, and C. Wetterich, Phys. Lett. B 727, 298 (2013).

[59] P. Donà, A. Eichhorn, and R. Percacci, Phys. Rev. D 89, 084035 (2014).

[60] R. Percacci and G. P. Vacca, Eur. Phys. J. C 75, 188 (2015).

[61] K.-y. Oda and M. Yamada, Classical Quantum Gravity 33, 125011 (2016).

[62] P. Donà, A. Eichhorn, P. Labus, and R. Percacci, Phys. Rev. D 93, 044049 (2016); 93, 129904(E) (2016).

[63] A. Eichhorn, A. Held, and J. M. Pawlowski, Phys. Rev. D 94, 104027 (2016).

[64] A. Eichhorn and S. Lippoldt, Phys. Lett. B 767, 142 (2017).

[65] N. Christiansen and A. Eichhorn, Phys. Lett. B 770, 154 (2017).

[66] A. Eichhorn and A. Held, Phys. Rev. D 96, 086025 (2017).

[67] J. Biemans, A. Platania, and F. Saueressig, J. High Energy Phys. 05 (2017) 093.

[68] A. Eichhorn and A. Held, Phys. Lett. B 777, 217 (2018).

[69] A. Eichhorn and F. Versteegen, J. High Energy Phys. 01 (2018) 030.

[70] A. Eichhorn, S. Lippoldt, and V. Skrinjar, Phys. Rev. D 97, 026002 (2018).

[71] M. Niedermaier and M. Reuter, Living Rev. Relativity 9, 5 (2006).

[72] R. Percacci, arXiv:0709.3851.

[73] D. F. Litim, Phil. Trans. R. Soc. A 369, 2759 (2011).

[74] M. Reuter and F. Saueressig, New J. Phys. 14, 055022 (2012).

[75] A. Bonanno and F. Saueressig, C.R. Phys. 18, 254 (2017).

[76] A. Eichhorn, arXiv:1709.03696.

[77] C. Wetterich, Phys. Lett. B 301, 90 (1993).

[78] U. Ellwanger, Z. Phys. C 62, 503 (1994).

[79] T. R. Morris, Int. J. Mod. Phys. A 09, 2411 (1994).

[80] D. F. Litim and J. M. Pawlowski, J. High Energy Phys. 09 (2002) 049.

[81] D. F. Litim and J. M. Pawlowski, Phys. Lett. B 546, 279 (2002).

[82] J. M. Pawlowski, arXiv:hep-th/0310018.

[83] J. M. Pawlowski, Ann. Phys. (Amsterdam) 322, 2831 (2007).

[84] M. Safari, Eur. Phys. J. C 76, 201 (2016).

[85] I. H. Bridle, J. A. Dietz, and T. R. Morris, J. High Energy Phys. 03 (2014) 093.

[86] J. A. Dietz and T. R. Morris, J. High Energy Phys. 04 (2015) 118.

[87] M. Safari and G. P. Vacca, J. High Energy Phys. 11 (2016) 139.

[88] C. M. Nieto, R. Percacci, and V. Skrinjar, Phys. Rev. D 96, 106019 (2017).

[89] J. Braun, H. Gies, and J. M. Pawlowski, Phys. Lett. B 684, 262 (2010).

[90] J. Braun, L. M. Haas, F. Marhauser, and J. M. Pawlowski, Phys. Rev. Lett. 106, 022002 (2011).

[91] J. Braun, A. Eichhorn, H. Gies, and J. M. Pawlowski, Eur. Phys. J. C 70, 689 (2010).

[92] L. Fister and J. M. Pawlowski, Phys. Rev. D 88, 045010 (2013).

[93] U. Reinosa, J. Serreau, M. Tissier, and N. Wschebor, Phys. Lett. B 742, 61 (2015).

[94] A. Bonanno and M. Reuter, Phys. Rev. D 65, 043508 (2002).
[95] A. Bonanno and M. Reuter, Phys. Lett. B 527, 9 (2002).

[96] E. Bentivegna, A. Bonanno, and M. Reuter, J. Cosmol. Astropart. Phys. 01 (2004) 001.

[97] M. Reuter and F. Saueressig, J. Cosmol. Astropart. Phys. 09 (2005) 012.

[98] A. Bonanno and M. Reuter, J. Cosmol. Astropart. Phys. 08 (2007) 024.

[99] S. Weinberg, Phys. Rev. D 81, 083535 (2010).

[100] A. Bonanno, Proc. Sci., CLAQG08 (2011) 008, [arXiv: 0911.2727].

[101] A. Bonanno and M. Reuter, Entropy 13, 274 (2011).

[102] B. Koch and I. Ramirez, Classical Quantum Gravity 28, 055008 (2011).

[103] R. Casadio, S. D. H. Hsu, and B. Mirza, Phys. Lett. B 695, 317 (2011).

[104] A. Contillo, Phys. Rev. D 83, 085016 (2011).

[105] A. Bonanno, A. Contillo, and R. Percacci, Classical Quantum Gravity 28, 145026 (2011).

[106] M. Hindmarsh, D. Litim, and C. Rahmede, J. Cosmol. Astropart. Phys. 07 (2011) 019.

[107] Y.-F. Cai and D. A. Easson, Phys. Rev. D 84, 103502 (2011).

[108] A. Bonanno, Phys. Rev. D 85, 081503 (2012).

[109] M. Hindmarsh and I. D. Saltas, Phys. Rev. D 86, 064029 (2012).

[110] A. Bonanno and M. Reuter, Phys. Rev. D 87, 084019 (2013).

[111] E. J. Copeland, C. Rahmede, and I. D. Saltas, Phys. Rev. D 91, 103530 (2015).

[112] D. Becker and M. Reuter, J. High Energy Phys. 12 (2014) 025.

[113] I. D. Saltas, J. Cosmol. Astropart. Phys. 02 (2016) 048.

[114] N. G. Nielsen, F. Sannino, and O. Svendsen, Phys. Rev. D 91, 103521 (2015).

[115] A. Bonanno and A. Platania, Phys. Lett. B 750, 638 (2015).

[116] K. Falls, D. F. Litim, K. Nikolakopoulos, and C. Rahmede, arXiv:1607.04962.

[117] A. Eichhorn, P. Labus, J. M. Pawlowski, and M. Reichert (to be published).

[118] N. Ohta, R. Percacci, and G. P. Vacca, Eur. Phys. J. C 76, 46 (2016).

[119] N. Ohta, R. Percacci, and G. P. Vacca, Phys. Rev. D 92 , 061501 (2015).

[120] C. S. Fischer, A. Maas, and J. M. Pawlowski, Ann. Phys. (Amsterdam) 324, 2408 (2009).

[121] C. S. Fischer and J. M. Pawlowski, Phys. Rev. D 80, 025023 (2009).

[122] R. Cuesta, K. Falls, and D. F. Litim (to be published).

[123] R. A. Cuesta Ramos, Ph.D. thesis, University of Sussex, 2016.

[124] J. M. Pawlowski and M. Reichert (to be published).

[125] D. Benedetti, New J. Phys. 14, 015005 (2012).

[126] K. Falls, J. High Energy Phys. 01 (2016) 069.

[127] J. A. M. Vermaseren, arXiv:math-ph/0010025.

[128] J. Kuipers, T. Ueda, J. A. M. Vermaseren, and J. Vollinga, Comput. Phys. Commun. 184, 1453 (2013).

[129] D. Brizuela, J. M. Martin-Garcia, and G. A. M. Marugan, Gen. Relativ. Gravit. 41, 2415 (2009).

[130] A. K. Cyrol, M. Mitter, and N. Strodthoff, Comput. Phys. Commun. 219, 346 (2017). 